\title{
Positively Curved Cubic Plane Graphs Are Finite
}

\author{
Liang Sun * \\ Department of Applied Mathematics \\ Beijing Institute of Technology \\ Beijing 100081, China \\ Xingxing $\mathrm{Yu}^{\dagger}$ \\ School of Mathematics \\ Georgia Institute of Technology \\ Atlanta, Georgia 30332 \\ yu@math.gatech.edu
}

\begin{abstract}
Let $G$ be an infinite plane graph such that $G$ is locally finite and every face of $G$ is bounded by a cycle. Then $G$ is said to be positively curved if, for every vertex $x$ of $G, 1-d(x) / 2+\Sigma_{x \in F} \frac{1}{|F|}>0$, where the summation is taken over all facial cycles $F$ containing $x$. Note that if $G$ is positively curved then the maximum degree of $G$ is at most 5. As a discrete analogue of a result in Riemannian geometry, Higuchi conjectured that if $G$ is positively curved then $G$ is finite. In this paper, we establish this conjecture for cubic graphs.
\end{abstract}

\footnotetext{
*Partially supported by a grant from China Scholarship Council

${ }^{\dagger}$ Partially supported by NSF grant DMS-9970527
} 


\section{Introduction}

The graphs considered in this paper are simple, but may be finite or infinite. Let $G$ be a graph. Then $V(G)$ and $E(G)$ denote the vertex set and edge set of $G$, respectively. We use $|G|$ to denote the number of vertices of $G$. For any $x \in V(G), d_{G}(x)$ denotes the number of edges of $G$ incident with $x$. (We use $d(x)$ if no confusion arises.) We say that $G$ is cubic if $d(x)=3$ for all $x \in V(G)$. We say that $G$ is locally finite if $d(x)$ is finite for all $x \in V(G)$. If there is no danger of confusion we write $x \in G$ in stead of $x \in V(G)$. A cycle in $G$ is a finite connected subgraph of $G$ in which every vertex has degree 2. For subgraphs $G$ and $H$ of a graph, we use $G \cup H$ and $G \cap H$ to denote the union and intersection of $G$ and $H$, respectively. For any graph $G$ and any $X \subset V(G)$, $G-X$ denote the graph obtained from $G$ by deleting $X$ and all edges of $G$ incident with $X$. If $G$ is connected and $G-X$ is not connected, then $X$ is called a vertex cut of $G$. If $X$ is a vertex cut of $G$ and $|X|=k$, then $X$ is called a $k$-cut.

A plane graph is a graph drawn in the plane with no pair of edges crossing. Let $G$ be a plane graph. Then the vertices and edges incident with a common face are called cofacial. We say that a face of $G$ is bounded by a cycle if the edges of $G$ incident with it induce a cycle in $G$, and such a cycle is called a facial cycle of $G$. Let $C$ be a cycle in a plane graph. Then we can speak of two orientations on $C$ : clockwise orientation and counter-clockwise orientation. Let $u, v$ be distinct vertices of $C$. We use $C[u, v]$ to denote the colckwise path in $C$ from $u$ to $v$. We use $C(u, v)$ to denote the graph obtained from $C[u, v]$ by deleting $u$ and $v$. We define $C[u, v)$ and $C(u, v]$ similarly.

In [2], the curvature of a plane graph is introduced as a discrete analogue of the sectional curvature of a Riemannian manifold, and a criterion is given for the hyperbolicity of a plane graph. For more detail, see [2] and the references in [2].

Let $G$ be a plane graph (finite or infinite) such that (1) $G$ is locally finite and (2) every face of $G$ is bounded by a cycle. Then the combinatorial curvature of $G$ is the function $\Phi_{G}: V(G) \rightarrow R$ such that for any $x \in V(G)$,

$$
\Phi_{G}(x)=1-\frac{d(x)}{2}+\Sigma_{x \in F} \frac{1}{|F|},
$$

where the summation is taken over all facial cycles of $G$ containing $x$. See Figure 1 for an example. If $\Phi_{G}(x)>0$ for all $x \in V(G)$, then we say that $G$ is positively curved.

As pointed in [2], $\Phi_{G}(x)$ may be interpreted as the degree of difficulty for tiling the plane at $x$, and it is dual to another curvature introduced by Gromov [1]. Higuchi in [2] proves that under some minor requirements, if $\Phi_{G}(x)<0$ for all $x \in V(G)$ then there exists a constant $\epsilon>0$ such that $\Phi_{G}(x)<-\epsilon$ for all $x \in V(G)$. This is then used to derive a discrete analogue of a fact in Riemannian geometry concernning isoperimetric inequalities. 




Figure 1: $\Phi_{G}(x)=1-3 / 2+(1 / 3+1 / 4+1 / 5)=17 / 60$

The following conjecture is posed in [2] which is a discrete analogue of a result of Myers [3]: a complete Riemannian manifold with Ricci curvature bounded below by a positive number is compact and has finite fundamental group.

(1.1) Conjecture. Let $G$ be a locally finite plane graph such that every face of $G$ is bounded by a cycle. If $\Phi_{G}(x)>0$ for all $x \in V(G)$ then $G$ is a finite graph.

The plane graph in Figure 2 is obtained from two vertex disjoint cycles $u_{1} u_{2} \ldots u_{n} u_{1}$ and $v_{1} v_{2} \ldots v_{n} v_{1}$ by adding a perfect matching $\left\{u_{i} v_{i}: i=1,2, \ldots, n\right\}$. It is easy to verify that this graph is positively curved. Hence, there are arbitrarily large positively curved graphs.

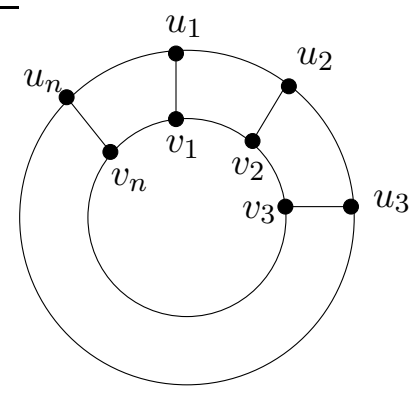

Figure 2: A positively curved graph.

Higuchi verified (1.1) for some special classes of graphs, and he noted that his method brings no insight to (1.1). Note that if $\Phi_{G}(x)>0$ for all $x \in V(G)$ then $d(x) \leq 5$ for all $x \in V(G)$.

The main result of this paper is the following which establishs (1.1) for all cubic graphs. We believe that our method offers a possible approach to establish (1.1) completely.

(1.2) Theorem. Let $G$ be a cubic plane graph such that every face of $G$ is bounded by a cycle. If $\Phi_{G}(x)>0$ for all $x \in V(G)$ then $G$ is a finite graph. 
The main idea of our proof is as follows. Assume that $G$ is infinite. First, we prove the existence of an infinite sequence of disjoint cycles $\left(C_{0}, C_{1}, \ldots\right)$ which captures a lot of structural information of $G$. This is done without requiring $G$ be cubic. We then assume that $G$ is positively curved, and derive a contradiction by showing that for all sufficiently large $n,\left|C_{n}\right|>\left|C_{n+1}\right|$. This is done through case analysis.

This paper is organized as follows. In Section 2, we show first how to produce an infinite sequence of cycles mentioned above. We then use that sequence to derive a plane embedding of the same graph with the same curvature function, but is easier to deal with. In Section 3, we introduce necessary notation and derive further structural information about positively curved cubic plane graphs. In Sections 4 through 7, we prove (1.2).

\section{Nice sequences}

The main objective of this section is to derive some useful structural information about infnite plane graphs. To this end, we need the following convenient concept.

(2.1) Definition. Let $H$ be a subgraph (finite or infinite) of a graph $G$ (finite or infinite). An $H$-bridge of $G$ is a subgraph of $G$ which is induced by either (1) an edge $e \in E(K)-$ $E(H)$ with both incident vertices on $H$, or (2) edges in a component $D$ of $G-V(H)$ and edges from $D$ to $H$. If $B$ is an $H$-bridge of $G$, then the vertices in $V(H) \cap V(B)$ are attachments of $B$ on $H$. Note that an $H$-bridge of $G$ may be infinite.

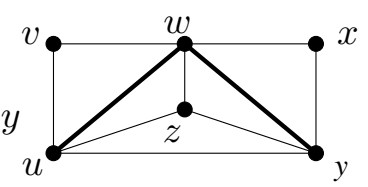

Figure 3: A path $H$ and its bridges

In Figure 3, $H=u w y$ is a path. The $H$-bridges of $G$ are the subgraphs induced by the following sets of edges: $\{u v, v w\},\{w x, x y\},\{z u, z w, z y\}$, and $\{u y\}$. Now we turn our attention to the description of a "nice" sequence of cycles.

Let $G$ be an infinite plane graph such that $G$ is locally finite and every face of $G$ is bounded by a cycle. Let $F$ be a facial cycle of $G$ and let $R(F)$ denote the closure of the face of $G$ bounded by $F$. (Thus, $F$ is the boundary of $R(F)$.) For any cycle $C$ in $G$, we define $R_{F}(C)$ as follows. By the Jordan curve theorem, $C$ divides the plane into two closed regions whose intersection is $C$. We use $R_{F}(C)$ to denote the closed region of the plane bounded by $C$ which contains $R(F)$. Hence, $R_{F}(F)=R(F)$. For any cycle $C$ in $G$, we use $G_{F}(C)$ to denote the subgraph of $G$ contained in $R_{F}(C)$. 
(2.2) Definition. Let $G$ be an infinite plane graph such that $G$ is locally finite and every face of $G$ is bounded by a cycle. Let $F$ be a facial cycle of $G$. A sequence of disjoint cycles $\left(C_{0}, C_{1}, \ldots\right)$ in $G$ is called a nice sequence starting with $F$ if the following conditions hold:

(1) $C_{0}=F$,

(2) for $i \geq 0, R_{F}\left(C_{i}\right) \subset R_{F}\left(C_{i+1}\right)$ (and hence, $G_{F}\left(C_{i}\right) \subset G_{F}\left(C_{i+1}\right)$ ),

(3) for $i \geq 0$, every $\left(G_{F}\left(C_{i}\right) \cup C_{i+1}\right)$-bridge of $G_{F}\left(C_{i+1}\right)$ has at most one attachment on $C_{i+1}$, and

(4) for $i \geq 0, G-V\left(G_{F}\left(C_{i}\right)\right)$ is infinite.

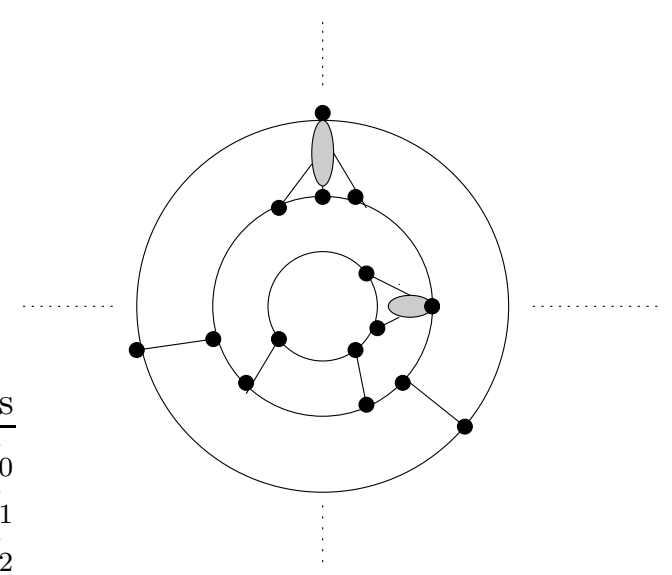

Figure 4: An example of a nice seqence

Figure 4 illustrates a nice sequence. The shaded regions represent subgraphs which are finite or infinite. Notice that a $\left(G_{F}\left(C_{i}\right) \cup C_{i+1}\right)$-bridge $B$ may be infinite, but the vertices and edges of $B$ cofacial with a vertex of $C_{i+1}$ form a finite subgraph $B^{*}$ of $G$. Because every face of $G$ is bounded by a cycle, $B^{*}$ is the union of two finite paths.

We are now ready to describe and prove the main result of this section.

(2.3) Theorem. Let $G$ be a connected infinite plane graph such that $G$ is locally finite and every face of $G$ is bounded by a cycle, and let $F$ be a facial cycle of $G$. Then $G$ has a nice sequence starting with $F$.

Proof. Let $C_{0}=F$, let $G_{F}\left(C_{0}\right)=C_{0}$, and let $R_{F}\left(C_{0}\right)$ be the closure of the face bounded by $F$. Suppose that we have constructed $\left(C_{0}, \cdots, C_{k}\right)$ for some $k \geq 0$ such that

(1) $C_{0}=F$, 
(2) for $0 \leq i \leq k-1, R_{F}\left(C_{i}\right) \subset R_{F}\left(C_{i+1}\right)$ and $G_{F}\left(C_{i}\right) \subset G_{F}\left(C_{i+1}\right)$,

(3) for $0 \leq i \leq k-1$, every $\left(G_{F}\left(C_{i}\right) \cup C_{i+1}\right)$-bridge of $G_{F}\left(C_{i+1}\right)$ has at most one attachment on $C_{i+1}$, and

(4) for $0 \leq i \leq k, G-G_{F}\left(C_{i}\right)$ is infinite.

The remainder of this proof shows how to construct the next cycle for the desired sequence. Consider the graph $H:=G-G_{F}\left(C_{k}\right)$. Note that $H$ need not be connected.

First, we claim that $H$ has only finitely many blocks. For, suppose $H$ has infinitely many blocks. Because $G$ is connected, every block of $H$ has a neighbor on $C_{k}$. Since $\left|C_{k}\right|$ is finite, some vertex of $C_{k}$ is adjacent to infinitely many blocks of $H$. This contradicts the assumption that $G$ is a locally finite.

Therefore, since $H$ is infinite, some block of $H$, say $B$, is infinite. Let $C_{k+1}$ denote the subgraph of $H$ consisting of vertices and edges of $B$ cofacial with a vertex of $C_{k}$. Observe that $C_{k+1}$ is finite because $C_{k}$ is finite, $G$ is locally finite, and every face of $G$ is bounded by a cycle. Since $B$ is 2-connected, $C_{k+1}$ is a cycle.

Obviously, $R_{F}\left(C_{k}\right) \subset R_{F}\left(C_{k+1}\right)$ and $G_{F}\left(C_{k}\right) \subset G_{F}\left(C_{k+1}\right)$. Because $B$ is a block of $G_{F}\left(C_{k}\right)$, every $\left(G_{F}\left(C_{k}\right) \cup C_{k+1}\right)$-bridge has at most one attachment on $C_{k+1}$. Because $B$ is infinite and $C_{k+1}$ is finite, $B-V\left(C_{k+1}\right)$ is infinite. Hence $G-V\left(G_{F}\left(C_{k+1}\right)\right)$ is infinite. So the sequence $\left(C_{0}, \ldots, C_{k+1}\right)$ statisies (1)-(4) above with $k+1$ replaceing $k$.

This process can be continued with $\left(C_{0}, \ldots, C_{k+1}\right)$ replacing $\left(C_{0}, \ldots, C_{k}\right)$. Hence, the desired nice sequence $\left(C_{0}, C_{1}, \ldots\right)$ exists.

To facilitate later discussions, we will work with a "nice" embedding of a plane graph $G$ which has the same combinatorial curvature as $G$.

(2.4) Theorem. Suppose that $G$ is an infinite locally finite plane graph with a facial cycle $F$, and let $\left(C_{0}, C_{1}, C_{2}, \cdots\right)$ be a nice sequence in $G$ starting with $F$. Then $G$ has an embedding $G^{\prime}$ in the plane such that

(1) $G_{F}^{\prime}\left(C_{i}\right)$ is contained in the closed disc bounded by $C_{i}$, and

(2) $G$ and $G^{\prime}$ have the same combinatorial curvature.

Proof. Consider the graphs $H_{i}:=G_{F}\left(C_{i+1}\right)-\left(G_{F}\left(C_{i}\right)-C_{i}\right)$ for all $i \geq 0$. Note that each $H_{i}$ is a subgaph of $G$. Hence, $H_{i}$ is a plane graph and both $C_{i}$ and $C_{i+1}$ are facial cycles of $H_{i}$. Therefore, $H_{i}$ has an embedding $H_{i}^{\prime}$ in the plane such that

(a) for any cycle $F$ of $H_{i}, F$ is a facial cycle of $H_{i}^{\prime}$ if, and only if $F$ is a facical cycle of $H_{i}$,

(b) the face of $H_{i}^{\prime}$ bounded by $C_{i}$ is an open disc in the plane,

(c) the face of $H_{i}^{\prime}$ bounded by $C_{i+1}$ is an unbounded region in the plane. 
of $G$.

By assembling the embeddings $H_{i}^{\prime}$, for all $i \geq 0$, we obtain the desired embedding $G^{\prime}$

We say that $G$ is nicely embedded with respect to a nice sequence $\left(C_{0}, C_{1}, \ldots\right)$ if, for each $i \geq 0, G_{F}\left(C_{i}\right)$ is contained in the closed disc bounded by $C_{i}$.

\section{Notation and convention}

Throughout this section, we assume that $G$ is a cubic infinite plane graph such that every face of $G$ is bounded by a cycle.

Let $v$ be a vertex of $G$. Let $F_{1}, F_{2}$ and $F_{3}$ be the facial cycles of $G$ containing $v$, and assume that $\left|F_{1}\right| \leq\left|F_{2}\right| \leq\left|F_{3}\right|$. We define $\ell(v)=\left(\left|F_{1}\right|,\left|F_{2}\right|,\left|F_{3}\right|\right)$. If $\left|F_{1}\right| \geq m_{1}$, $\left|F_{2}\right| \geq m_{2}$, and $\left|F_{3}\right| \geq m_{3}$, then we write $\ell(v) \geq\left(m_{1}, m_{2}, m_{3}\right)$.

For a vertex $v$ of $G$ with $\ell(v)=\left(m_{1}, m_{2}, m_{3}\right), \Phi_{G}(v)>0$ iff $1 / m_{1}+1 / m_{2}+1 / m_{3}>$ $1 / 2$. The following is a partial list of triples $\left(m_{1}, m_{2}, m_{3}\right)$ such that $1 / m_{1}+1 / m_{2}+1 / m_{3} \leq$ $1 / 2$.

(3.1) Lemma. Let $G$ be a cubic infinite plane graph such that every face of $G$ is bounded by a cycle and let $v \in V(G)$. Let

$\mathcal{T}=\{(3,10,15),(3,11,14),(3,12,12),(4,6,12),(4,7,10),(4,8,8),(5,5,10),(5,6,8),(5,7,7),(6,6,6)\}$

. If $\ell(v) \in \mathcal{T}$, then $\Phi_{G}(v) \leq 0$.

Throughout the rest of the paper, we will derive contradictions to (3.1) by showing that in a positively curved cubic infinite plane graph there is a vertex $v$ such that $\ell(v) \in \mathcal{T}$. For ease of presentation, we will not refer to (3.1) explicitely.

Let $F$ be a facial cycle of $G$. By $(2.3), G$ has a nice sequence $\left(C_{0}, C_{1}, \ldots\right)$ starting with $F$ and satisfying (1)-(4) of (2.2). By (2.4), we may assume that $G$ is nicely embedded with respect to $\left(C_{0}, C_{2}, \ldots\right)$.

A vertex $v$ of $G$ is called an in-vertex (respectively, out-vertex) if $v \in C_{i}$ for some $i \geq 0$ and $v$ is incident with an edge contained in the annulus region between $C_{i}$ and $C_{i-1}$ (respectively, $C_{i+1}$ ). If $v$ is an in-vertex on $C_{i}$, then we use $A(v), L(v), R(v)$ to denote the facial cycles of $G$ containing $v$, where $A(v)$ is between $C_{i}$ and $C_{i+1}$ and $A(v), R(v), L(v)$ occur in this clockwise order around $v$. (We can think of $L(v)$ as to the left of $v$, and $R(v)$ as to the right of $v$.) If $w$ is an out-vertex on $C_{i}$, then we use $B(w), L(w), R(w)$ to denote the facial cycles of $G$ containing $w$, where $B(w)$ is between $C_{i}$ and $C_{i-1}$ and $B(w), L(w), R(w)$ occur around $w$ in that clockwise order. (Again, we can think of $L(w)$ as to the left of $w$, and $R(w)$ as to the right of $w$.) See Figure 5.

By the choice of $\left(C_{0}, C_{1}, \ldots\right)$, we have the following two observations which will be used frequently (often without explicit reference). 

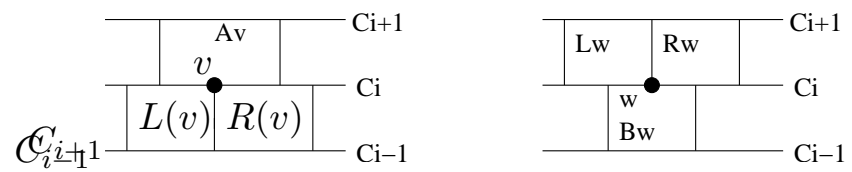

Figure 5: $v$ is an in-vertex on $C_{i}$ and $w$ is an out-vertex on $C_{i}$

(3.2) Lemma. Let $G$ be a cubic infinite plane graph with a nice sequence $\left(C_{0}, C_{1}, \ldots\right)$ and assume that $G$ is nicely embedded with respect to $\left(C_{0}, C_{1}, \ldots\right)$. For any in-vertex $v$ on $C_{i},|L(v)| \geq 4 \leq|R(v)|$. For any out-vertex $w$ on $C_{i},|B(w)| \geq 5$.

(3.3) Lemma. Let $G$ be a cubic infinite plane graph with a nice sequence $\left(C_{0}, C_{1}, \ldots\right)$ and assume that $G$ is nicely embedded with respect to $\left(C_{0}, C_{1}, \ldots\right)$. For any facial cycle $F$ of length $4,\left|F \cap C_{i}\right| \neq 1$.

The next lemma allows us to get rid of certain 2-cuts of $G$ in the proof of (1.2).

(3.4) Lemma. If there is a cubic infinite plane graph with positive combinatorial curvature, then there is a cubic infnite plane graph $G$ with positive combinatorial curvature such that

(1) $G$ has a nice sequence $\left(C_{0}, C_{1}, \ldots\right)$, and

(2) for any $k \geq 1$ and for any 2-cut $T$ of $G$ contained in $V\left(C_{k}\right), \bigcup_{0 \leq i \leq k-1} C_{i}$ and $\bigcup_{i \geq k+1} C_{i}$ belong to different components of $G-T$.

Proof. Let $G$ be a cubic infinite plane graph such that $G$ is locally finite and every face of $G$ is bounded by a cycle. Assume that $G$ has positive combinatorial curvature. So for any $x \in V(G), \Phi_{G}(x)>0$. Suppose that there is some $k \geq 1$ and a 2-cut $T=\{u, v\}$ of $G$ contained in $V\left(C_{k}\right)$ such that $\bigcup_{0 \leq i \leq k-1} C_{i}$ and $\bigcup_{i \geq k+1} C_{i}$ belong to the same component of $G-T$. Let $B$ denote the $T$-bridge of $G$ not containing $\bigcup_{i \neq k} C_{i}$. We may assume that $T$ is chosen so that $B$ is maximal. Then $B$ contains at least two neighbors of $u$ and at least two neighbors of $v$. So let $H$ denote the plane graph obtained from $G$ by replacing $B$ with the edge $u v$. Then $H$ is a cubic infinite plane graph. Moreover, for each vertex $x$ of $H$, the length of any facical cycle of $H$ containing $x$ is not longer than the corresponding facial cycle of $G$. So $H$ is also positively curved. Since for all $k \geq 1,\left|C_{k}\right|$ is finite, there are only finitely many 2 -cuts contained in $V\left(C_{k}\right)$. So we can repeatedly perform the above operation to eliminate all 2-cuts cotained in $V\left(C_{k}\right)$ not satisfying (2). We can deal with $C_{0}, C_{1}, C_{2}, \ldots$ in that order, and we see that (3.4) holds.

The proof of (1.2) is divided into the following stages. Assume that $G$ is a cubic infinite infinite plane graph. Then by the results in Section $2, G$ has a nice sequence 
$\left(C_{0}, C_{2}, \ldots\right)$ and we can assume that $G$ is nicely embedded with respect to that sequence. First, we show that for all sufficiently large $i$, there are at most three vertices of $C_{i}$ between any two consecutive in-vertices on $C_{i}$. This is done in Section 4 . We then use the result in Section 4 to show that for all sufficiently large $i$, there are at most two vertices of $C_{i}$ between any two consecutive in-vertices on $C_{i}$, and this is done in Section 5. In Section 6, we further show that for all sufficiently large $i$, there are at most one vertex of $C_{i}$ between any two consecutive in-vertices on $C_{i}$. Finally we complete the proof in Section 7 by showing that for all sufficiently large $i,\left|C_{j}\right|>\left|C_{i}\right|$ for all $j>i$.

\section{Four vertices between consecutive in-vertices}

For convenience, we assume throughout this section that $G$ is a positively curved cubic infinite plane graph which is locally finite and whose faces are bounded by cycles. Moreover, $G$ has a nice sequence $\left(C_{0}, C_{1}, \ldots\right)$ and $G$ is nicely embedded with respect to $\left(C_{0}, C_{1}, \ldots\right)$.

The main result of this section is the following: if $i$ is large enough, then there are at most three vertices of $C_{i}$ between any two consecutive in-vertices on $C_{i}$. This is done through a series of lemmas. For the statement and proof of the first lemma, we refer to Figure 6.

(4.1) Lemma. Let $i \geq 3$, and let $a_{1}$ and $a_{2}$ be consecutive in-vertices on $C_{i}$ such that (i) $R\left(a_{1}\right)=L\left(a_{2}\right)$ and (ii) $\left|C_{i}\left(a_{1}, a_{2}\right)\right| \geq 4$. Let $b_{1}, b_{2}$ be the out-vertices on $C_{i-1}$ such that $R\left(b_{1}\right)=L\left(b_{2}\right)=R\left(a_{1}\right)$. If $C_{i-1}\left(b_{1}, b_{2}\right)=\emptyset$, then $\left|L\left(b_{1}\right)\right|=\left|R\left(b_{2}\right)\right|=3$.

Proof. Suppose $C_{i-1}\left(b_{1}, b_{2}\right)=\emptyset$. Since $G$ is cubic, $B\left(b_{1}\right)=B\left(b_{2}\right)$. So let $c_{1}, c_{2}$ be the out-vertices on $C_{i-2}$ such that $R\left(c_{1}\right)=B\left(b_{1}\right)=L\left(c_{2}\right)$. By (ii), $\left|R\left(b_{1}\right)\right|=\left|L\left(b_{2}\right)\right| \geq 8$. Since $C_{i-1}\left(b_{1}, b_{2}\right)=\emptyset,\left|B\left(b_{1}\right)\right|=\left|B\left(b_{2}\right)\right| \geq 6$.

Therefore, $\left|L\left(b_{1}\right)\right| \leq 4$ and $\left|R\left(b_{2}\right)\right| \leq 4$; for otherwise, $\ell\left(b_{1}\right) \geq(5,6,8)$ or $\ell\left(b_{2}\right) \geq$ $(5,6,8)$, a contradiction. Hence, we consider two cases.

Case 1. $\left|L\left(b_{1}\right)\right|=\left|R\left(b_{2}\right)\right|=4$.

By (3.3), $\left|L\left(b_{1}\right) \cap C_{i-1}\right| \in\{2,3\}$ and $\left|R\left(b_{2}\right) \cap C_{i-1}\right| \in\{2,3\}$.

First, assume that $\left|L\left(b_{1}\right) \cap C_{i-1}\right|=2=\left|R\left(b_{2}\right) \cap C_{i-1}\right|$. Since $G$ is cubic and $C_{i-1}\left(b_{1}, b_{2}\right)=\emptyset,\left|B\left(b_{1}\right)\right| \geq 8$. Hence $\ell\left(b_{1}\right) \geq(4,8,8)$, a contradiction.

Now assume that $\left|L\left(b_{1}\right) \cap C_{i-1}\right|=2$ and $\left|R\left(b_{2}\right) \cap C_{i-1}\right| \neq 2$ or $\left|L\left(b_{1}\right) \cap C_{i-1}\right| \neq 2$ and $\left|R\left(b_{2}\right) \cap C_{i-1}\right|=2$. By symmetry, assume the former. See Figure 6(a). Then $\left|R\left(b_{1}\right)\right|=\left|L\left(b_{2}\right)\right| \geq 9$, and since $G$ is cubic and $C_{i-1}\left(b_{1}, b_{2}\right)=\emptyset,\left|B\left(b_{1}\right)\right|=\left|B\left(b_{2}\right)\right| \geq 7$. Thus, $\ell\left(b_{1}\right)=\ell\left(b_{2}\right)=(4,7,9)$, for otherwise, there would exist $i \in\{1,2\}$ such that $\ell\left(b_{i}\right) \geq(5,7,9)$ or $(4,8,9)$ or $(4,7,10)$. Hence, $C_{i-2}\left(c_{1}, c_{2}\right)=\emptyset$ and $c_{2}$ is adjacent to $C_{i-1}$. So $\left|B\left(c_{2}\right)\right| \geq 6$ and $\left|R\left(c_{2}\right)\right| \geq 5$ (because $\left|R\left(b_{2}\right) \cap C_{i-1}\right|=3$ ). If $\left|R\left(c_{2}\right)\right|=5$ 
then $\left|B\left(c_{2}\right)\right| \geq 7$, and hence, $\ell\left(c_{2}\right) \geq(5,7,7)$, a contradiction. So $\left|R\left(c_{2}\right)\right| \geq 6$. Then $\ell\left(c_{2}\right) \geq(6,6,7)$, a contradiction.

So $\left|L\left(b_{1}\right) \cap C_{i-1}\right|=3=\left|R\left(b_{2}\right) \cap C_{i-1}\right|$. See Figure 6(b). Hence, $\left|R\left(b_{1}\right)\right|=\left|L\left(b_{2}\right)\right| \geq 10$. Since $\left|B\left(b_{1}\right)\right|=\left|B\left(b_{2}\right)\right| \geq 6, \ell\left(b_{1}\right) \geq(4,6,10)$ and $\ell\left(b_{2}\right) \geq(4,6,10)$. In fact, $\left|B\left(b_{1}\right)\right|=$ $\left|B\left(b_{2}\right)\right|=6$, as otherwise $\ell\left(b_{1}\right) \geq(4,7,10)$ or $\ell\left(b_{2}\right) \geq(4,7,10)$. So $C_{i-2}\left(c_{1}, c_{2}\right)=\emptyset$, $\left|L\left(c_{1}\right)\right| \geq 5 \leq\left|R\left(c_{2}\right)\right|$, and $\left|B\left(c_{1}\right)\right|=\left|B\left(c_{2}\right)\right| \geq 6$. Suppose $\left|L\left(c_{1}\right)\right|=\left|R\left(c_{2}\right)\right|=5$. Then $\left|L\left(c_{1}\right) \cap C_{i-2}\right|=2=\left|R\left(c_{2}\right) \cap C_{i-2}\right|$. Since $G$ is 2-connected and $C_{i-2}\left(c_{1}, c_{2}\right)=\emptyset$, $\left|B\left(c_{1}\right)\right|=\left|B\left(c_{2}\right)\right| \geq 8$. Hence $\ell\left(c_{1}\right) \geq(5,6,8)$, a contradiction. So $\left|L\left(c_{1}\right)\right| \geq 6$ or $\left|R\left(c_{2}\right)\right| \geq 6$. Then $\ell\left(c_{1}\right) \geq(6,6,6)$ or $\ell\left(c_{2}\right) \geq(6,6,6)$, a contradiction.

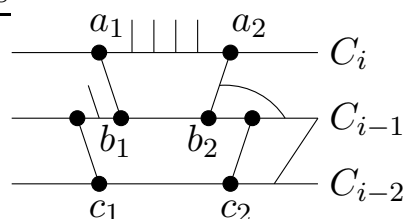

(a)

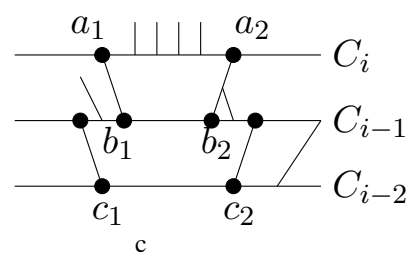

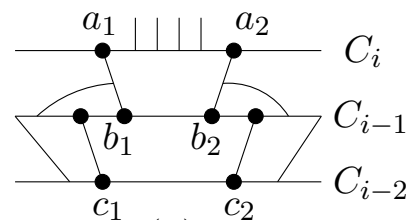

(b)

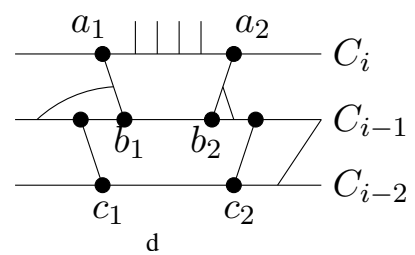

Figure 6: Proof of Lemma (4.1)

Case 2. $\left|L\left(b_{1}\right)\right|=4$ and $\left|R\left(b_{2}\right)\right|=3$, or $\left|L\left(b_{1}\right)\right|=3$ and $\left|R\left(b_{2}\right)\right|=4$.

By symmetry, we may assume the former. Since $\left|R\left(b_{2}\right)\right|=3$ and $C_{i-1}\left(b_{1}, b_{2}\right)=\emptyset$, $\left|L\left(b_{2}\right)\right|=\left|R\left(b_{1}\right)\right| \geq 9$ and $\left|B\left(b_{1}\right)\right|=\left|B\left(b_{2}\right)\right| \geq 7$.

First, assume that $\left|L\left(b_{1}\right) \cap C_{i-1}\right|=2$. See Figure 6(c). Then since $G$ is cubic and $C_{i-1}\left(b_{1}, b_{2}\right)=\emptyset,\left|B\left(b_{1}\right)\right| \geq 8$. Therefore, $\ell\left(b_{1}\right) \geq(4,8,9)$, a contradiction.

So $\left|L\left(b_{1}\right) \cap C_{i-1}\right|=3$. See Figure $6(\mathrm{~d})$. Then $\left|B\left(b_{1}\right)\right| \geq 7$ and $\left|R\left(b_{1}\right)\right| \geq 10$. So $\ell\left(b_{1}\right) \geq(4,7,10)$, a contradiction.

(4.2) Lemma. Let $i \geq 5$, and let $a_{1}$ and $a_{2}$ be consecutive in-vertices on $C_{i}$ such that (i) $R\left(a_{1}\right)=L\left(a_{2}\right)$ and (ii) $\left|C_{i}\left(a_{1}, a_{2}\right)\right| \geq 4$. Let $b_{1}$ and $b_{2}$ be the out-vertices on $C_{i-1}$ such that $R\left(b_{1}\right)=L\left(b_{2}\right)=R\left(a_{1}\right)$. Then

(1) $\left|C_{i-1}\left(b_{1}, b_{2}\right)\right| \neq 1$, and

(2) $\left|C_{i-1}\left(b_{1}, b_{2}\right)\right| \neq 2$ if $i \geq 7$. 
Proof. (1) Suppose $\left|C_{i-1}\left(b_{1}, b_{2}\right)\right|=1$. Let $b$ be the only vertex in $C_{i-1}\left(b_{1}, b_{2}\right)$. Since $G$ is 2-connected, $b$ is an in-vertex and $B\left(b_{1}\right)=L(b)$. Let $c_{1}, c, c_{2}$ be the out-vertices on $C_{i-2}$ such that $R\left(c_{1}\right)=L(c)=B\left(b_{1}\right)$ and $L\left(c_{2}\right)=B\left(b_{2}\right)$. See Figure 7 .

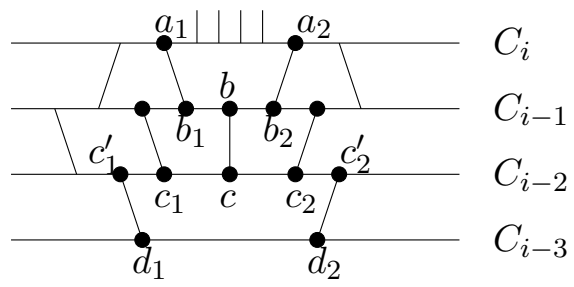

Figure 7: $C_{i-1}\left(b_{1}, b_{2}\right) \mid=1$.

Note that $\left|R\left(b_{1}\right)\right|=|A(b)|=\left|L\left(b_{2}\right)\right| \geq 9,|L(b)|=\left|B\left(b_{1}\right)\right| \geq 5$, and $|R(b)|=\left|B\left(b_{2}\right)\right| \geq$ 5. Hence $\ell(b) \geq(5,5,9)$. Therefore, $|L(b)|=|R(b)|=5$ and $|A(b)|=9$; otherwise, $\ell(b) \geq(5,6,9)$ or $(5,5,10)$, a contradiction.

Since $|L(b)|=|R(b)|=5, C_{i-2}\left(c_{1}, c\right)=\emptyset=C_{i-2}\left(c, c_{2}\right), b$ is adjacent to $c$, and both $c_{1}$ and $c_{2}$ are adjacent to $C_{i-1}$. Therefore, $\left|B\left(c_{1}\right)\right|=|B(c)|=\left|B\left(c_{2}\right)\right| \geq 7$ and $\left|L\left(b_{1}\right)\right| \geq 5 \leq\left|R\left(b_{2}\right)\right|$. So $\left|L\left(b_{1}\right)\right|=5=\left|R\left(b_{2}\right)\right|$, for otherwise, $\ell\left(b_{1}\right) \geq(5,6,9)$ or $\ell\left(b_{2}\right) \geq(5,6,9)$, a contradiction.

Since $G$ is 2-connected, let $c_{1}^{\prime}, c_{2}^{\prime}$ be in-vertices on $C_{i-2}$ such that $R\left(c_{1}^{\prime}\right)=L\left(c_{2}^{\prime}\right)$, and let $d_{1}, d_{2}$ be the out-vertices on $C_{i-3}$ such that $R\left(d_{1}\right)=L\left(d_{2}\right)=B\left(c_{1}\right)$.

We claim that $C_{i-2}\left(c_{1}^{\prime}, c_{1}\right)=\emptyset$ or $C_{i-2}\left(c_{2}, c_{2}^{\prime}\right)=\emptyset$. Otherwise, $\left|C_{i-2}\left(c_{1}^{\prime}, c_{2}^{\prime}\right)\right| \geq 5$. Since $i-2 \geq 3$, it follows from (4.1) that $\left|L\left(d_{1}\right)\right|=3=\left|R\left(d_{2}\right)\right|$ or $C_{i-1}\left(d_{1}, d_{2}\right) \neq \emptyset$. So $|B(c)| \geq 10$, and hence $\ell(c) \geq(5,5,10)$, a contradiction.

So by symmetry, assume that $C_{i-2}\left(c_{1}^{\prime}, c_{1}\right)=\emptyset$. Since $\left|L\left(b_{1}\right)\right|=5$ and $a_{1}$ is adjacent to $b_{1}$, we have $\left|A\left(c_{1}^{\prime}\right)\right| \geq 6$. So $\left|L\left(c_{1}\right)\right|=\left|A\left(c_{1}^{\prime}\right)\right|=6$ and $\left|B\left(c_{1}\right)\right|=7$, as otherwise, we would have $\ell\left(c_{1}\right) \geq(5,6,8)$ or $(5,7,7)$. Thus $c_{1}^{\prime}$ is adjacent to $d_{1}, C_{i-3}\left(d_{1}, d_{2}\right)=\emptyset$, and $\left|L\left(d_{1}\right)\right| \geq 5$. Moreover, if $\left|L\left(d_{1}\right)\right|=5$ then $\left|B\left(d_{1}\right)\right| \geq 7$. So $\ell\left(d_{1}\right) \geq(5,7,7)$ or $(6,6,7)$, a contradiction.

(2) Now suppose $i \geq 7$ and $\left|C_{i-1}\left(b_{1}, b_{2}\right)\right|=2$. Let $b_{3}, b_{4}$ denote the vertices in $C_{i-1}\left(b_{1}, b_{2}\right)$. By $(3.4)$, both $b_{3}$ and $b_{4}$ are in-vertices. Without loss of generality, we may assume that $R\left(b_{3}\right)=L\left(b_{4}\right)$. See Figure 8 .

Observe that $\left|R\left(b_{1}\right)\right|=\left|L\left(b_{2}\right)\right|=\left|A\left(b_{3}\right)\right|=\left|A\left(b_{4}\right)\right| \geq 10,\left|B\left(b_{1}\right)\right|=\left|L\left(b_{3}\right)\right| \geq 5 \leq$ $\left|R\left(b_{4}\right)\right|=\left|B\left(b_{2}\right)\right|,\left|R\left(b_{3}\right)\right|=\left|L\left(b_{4}\right)\right| \geq 4$. Furthermore, $\left|R\left(b_{3}\right)\right|=\left|L\left(b_{4}\right)\right|=4$ and $\left|L\left(b_{3}\right)\right|=\left|B\left(b_{1}\right)\right| \leq 6 \geq\left|R\left(b_{4}\right)\right|=\left|B\left(b_{2}\right)\right|$, for otherwise, there would exist $i \in\{3,4\}$ such that $\ell\left(b_{i}\right) \geq(5,5,10)$ or $(4,7,10)$, a contradiction.

So let $c_{3}, c_{4} \in V\left(C_{i-3}\right)$ such that $b_{3} c_{3}, b_{4} c_{4} \in E(G)$. Because $G$ is cubic and 2connected, $C_{i-2}\left(c_{4}, c_{3}\right)$ has at least two in-vertices. We claim that $C_{i-2}\left(c_{4}, c_{3}\right)$ also 

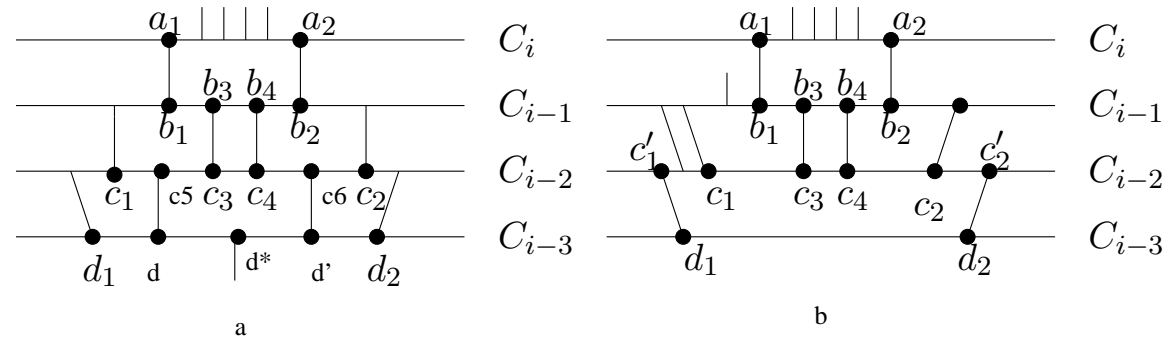

Figure 8: $i \geq 7$ and $\left|C_{i-1}\left(b_{1}, b_{2}\right)\right|=2$.

contains at least two out-vertices. For otherwise, $C_{i-2}\left(c_{4}, c_{3}\right)$ contains at most one outvertex. Because $\left|L\left(b_{3}\right)\right| \leq 6, C_{i-2}\left(c_{4}, c_{3}\right)$ contains exactly one out-vertex. So $\left|L\left(b_{3}\right)\right|=$ $6=\left|R\left(b_{4}\right)\right|$, and $\left|L\left(b_{1}\right)\right| \geq 5$. Thus $\ell\left(b_{1}\right) \geq(5,6,10)$, a contradiction. So let $c_{1}, c_{2}$ be distinct out-vertices on $C_{i-2}$ such that $R\left(c_{1}\right)=B\left(b_{1}\right)$ and $L\left(c_{2}\right)=B\left(b_{2}\right)$.

We claim that $C_{i-2}\left(c_{1}, c_{3}\right)=\emptyset=C_{i-2}\left(c_{4}, c_{2}\right)$. For otherwise, assume $C_{i-2}\left(c_{1}, c_{3}\right) \neq \emptyset$. See Figure $8(\mathrm{a})$. Because $\left|L\left(b_{3}\right)\right| \leq 6, C_{i-2}\left(c_{1}, c_{3}\right)$ consists of only one vertex, say $c_{5}$. Since $G$ is 2-connected, $c_{5}$ is an in-vertex. Thus we see that $\left|A\left(c_{5}\right)\right|=6,\left|L\left(c_{5}\right)\right| \geq 5$, and $\left|R\left(c_{5}\right)\right| \geq 6$. So $\left|L\left(c_{5}\right)\right|=5$ and $\left|R\left(c_{5}\right)\right| \leq 7$, as otherwise $\ell\left(c_{5}\right) \geq(5,6,8)$ or $(6,6,6)$. Hence $c_{5}$ is adjacent to a vertex, say $d$, on $C_{i-3}$. Assume that $C_{i-2}\left(c_{4}, c_{2}\right)=\emptyset$, then $\left|R\left(c_{5}\right)\right| \geq 7$. Thus $\left|R\left(c_{5}\right)\right|=7$, and hence $\left|R\left(c_{5}\right) \cap C_{i-3}\right|=2$. Therefore, since $\left|L\left(c_{5}\right)\right|=$ $5,|B(d)| \geq 7$. So $\ell(d) \geq(5,7,7)$, a contradiction. Thus $C_{i-2}\left(c_{4}, c_{2}\right) \neq \emptyset$. Because $\left|R\left(b_{4}\right)\right| \leq 6, C_{i-2}\left(c_{4}, c_{2}\right)$ consists of only one vertex, say $c_{6}$. Since $G$ is 2 -connected, $c_{6}$ is an in-vertex. Note that $\left|R\left(c_{6}\right)\right|=5$, for otherwise $\ell\left(c_{6}\right) \geq(6,6,6)$. Thus $c_{6}$ is adjacent to a vertex, say $d^{\prime}$, on $C_{i-3}$. If $C_{i-3}\left(d, d^{\prime}\right)=\emptyset$, then since $\left|L\left(c_{5}\right)\right|=\left|R\left(c_{6}\right)\right|=5,|B(d)| \geq 8$ and we would have $\ell(d) \geq(5,6,8)$. So $C_{i-3}\left(d, d^{\prime}\right) \neq \emptyset$. Since $\left|R\left(c_{5}\right)\right| \leq 7, C_{i-3}\left(d, d^{\prime}\right)$ contains only one vertex, say $d^{*}$. Because $\left|L\left(c_{5}\right)\right|=5=\left|R\left(c_{6}\right)\right|,\left|L\left(d^{*}\right)\right| \geq 6 \leq\left|R\left(d^{*}\right)\right|$. So $\ell\left(d^{*}\right) \geq(6,6,7)$, a contradiction.

Let $c_{1}^{\prime}, c_{2}^{\prime}$ denote the in-vertices on $C_{i-2}$ such that $R\left(c_{1}^{\prime}\right)=L\left(c_{2}^{\prime}\right)=B\left(c_{1}\right)$, and let $d_{1}, d_{2}$ be the out-vertices on $C_{i-3}$ such that $L\left(d_{1}\right)=R\left(d_{2}\right)=B\left(c_{1}\right)$. See Figure $8(\mathrm{~b})$. Since $i-2 \geq 5$ and $\left|C_{i-2}\left(c_{1}^{\prime} c_{2}^{\prime}\right)\right| \geq 4$, it follows from (1) that $\left|C_{i-3}\left(d_{1}, d_{2}\right)\right| \neq 1$. So by (4.1), $\left|L\left(d_{1}\right)\right|=\left|R\left(d_{2}\right)\right|=3$ or $\left|C_{i-3}\left(d_{1}, d_{2}\right)\right| \geq 2$. Thus $\left|B\left(c_{1}\right)\right|=\left|B\left(c_{2}\right)\right| \geq 10$. Since $\left|R\left(c_{1}\right)\right| \geq 5 \leq\left|L\left(c_{2}\right)\right|,\left|L\left(c_{1}\right)\right| \leq 4 \geq\left|R\left(c_{2}\right)\right|$ (or else there would exist $i \in\{1,2\}$ such that $\left.\ell\left(c_{i}\right) \geq(5,5,10)\right)$. Note $\left|R\left(b_{1}\right)\right|=\left|L\left(b_{2}\right)\right| \geq 10$ and $\left|B\left(b_{1}\right)\right| \geq 5 \leq\left|B\left(b_{2}\right)\right|$. So $\left|L\left(b_{1}\right)\right| \leq$ $4 \geq\left|R\left(b_{2}\right)\right|$ (otherwise there would exist some $i \in\{1,2\}$ such that $\ell\left(b_{i}\right) \geq(5,5,10)$ ).

We claim that $\left|L\left(b_{1}\right)\right|=4$ and $\left|L\left(b_{1}\right) \cap C_{i-1}\right|=2$ or $\left|R\left(b_{2}\right)\right|=4$ and $\left|R\left(b_{2}\right) \cap C_{i-1}\right|=2$. For otherwise, $\left|R\left(b_{1}\right)\right|=\left|L\left(b_{2}\right)\right| \geq 12$. Then $\left|L\left(b_{3}\right)\right|=\left|B\left(b_{1}\right)\right|=5$, or else $\ell\left(b_{3}\right) \geq$ $(4,6,12)$. Hence $\left|L\left(b_{1}\right)\right|=4$ and $\left|L\left(b_{1}\right) \cap C_{i-1}\right|=3$, and so, $\left|L\left(c_{1}\right)\right| \geq 5$, a contradiction.

Without loss of generality assume that $\left|L\left(b_{1}\right)\right|=4$ and $\left|L\left(b_{1}\right) \cap C_{i-1}\right|=2$. See Figure 
8(b). Then $\left|B\left(b_{1}\right)\right| \geq 6$. In fact, $\left|B\left(b_{1}\right)\right|=6$; otherwise, $\ell\left(b_{1}\right) \geq(4,7,10)$, a contradiction.

So $\left|L\left(c_{1}\right)\right|=4$ (otherwise $\ell\left(c_{1}\right) \geq(5,6,8)$ ). Hence $\left|L\left(c_{1}\right) \cap C_{i-2}\right|=2$ and $\left|B\left(c_{1}\right)\right| \geq 11$. In fact, $\left|B\left(c_{1}\right)\right|=11$ as otherwise $\ell\left(c_{1}\right) \geq(4,6,12)$. So $c_{1}^{\prime}$ is adjacent to $L\left(c_{1}\right)$ and $C_{i-2}\left(c_{2}, c_{2}^{\prime}\right)=\emptyset$. Thus $\left|A\left(c_{1}^{\prime}\right)\right| \geq 5,\left|A\left(c_{2}^{\prime}\right)\right|=\left|R\left(c_{2}\right)\right|=4$, and $\left|A\left(c_{2}^{\prime}\right) \cap C_{i-2}\right|=3$.

If $C_{i-3}\left(d_{1}, d_{2}\right) \neq \emptyset$, then since $i-2 \geq 5$ and by (1), $\left|C_{i-3}\left(d_{1}, d_{2}\right)\right| \geq 2$. Thus $\left|B\left(c_{1}\right)\right|=11$ implies that $c_{2}^{\prime}$ is adjacent to $d_{2}$. Since $\left|A\left(c_{2}^{\prime}\right)\right|=4,\left|R\left(d_{2}\right)\right| \geq 5$. Hence $\ell\left(d_{2}\right) \geq(5,5,11)$, a contradiction. Therefore, $C_{i-3}\left(d_{1}, d_{2}\right)=\emptyset$. Then by $(4.1),\left|L\left(d_{1}\right)\right|=$ 3 , and hence $\left|L\left(c_{1}^{\prime}\right)\right| \geq 5$. But now $\ell\left(c_{1}^{\prime}\right) \geq(5,5,11)$, a contradiction.

(4.3) Lemma. Let $i \geq 7$, let $a_{1}$ and $a_{2}$ be consecutive in-vertices on $C_{i}$ such that (i) $R\left(a_{1}\right)=L\left(a_{2}\right)$ and (ii) $\left|C_{i}\left(a_{1}, a_{2}\right)\right| \geq 4$. Let $b_{1}$ and $b_{2}$ be the out-vertices on $C_{i-1}$ such that $R\left(b_{1}\right)=L\left(b_{2}\right)=R\left(a_{1}\right)$. Assume that $C_{i-1}\left(b_{1}, b_{2}\right)=\emptyset$, and let $c_{1}, c_{2}$ denote the out-vertices on $C_{i-2}$ such that $R\left(c_{1}\right)=L\left(c_{2}\right)=B\left(b_{1}\right)=B\left(b_{2}\right)$. Then $C_{i-2}\left(c_{1}, c_{2}\right) \neq \emptyset$.

Proof. Suppose for a contradiction that $C_{i-2}\left(c_{1}, c_{2}\right)=\emptyset$. Since $G$ is 2-connected and cubic, let $b_{1}^{\prime}, b_{2}^{\prime}$ denote the consecutive in-vertices on $C_{i-1}$ such that $R\left(b_{1}^{\prime}\right)=L\left(b_{2}^{\prime}\right)=$ $B\left(b_{1}\right)=B\left(b_{2}\right)$; let $c_{1}^{\prime}, c_{2}^{\prime}$ be the consecutive in-vertices on $C_{i-2}$ such that $R\left(c_{1}^{\prime}\right)=L\left(c_{2}^{\prime}\right)=$ $B\left(c_{1}\right)=B\left(c_{2}\right)$; and let $d_{1}, d_{2}$ denote the out-vertices on $C_{i-3}$ such that $R\left(d_{1}\right)=L\left(d_{2}\right)=$ $B\left(c_{1}\right)$. See Figure 9 .

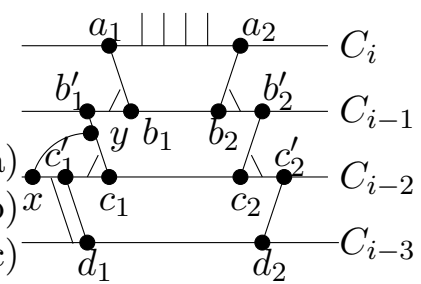

Figure 9: Proof of Lemma (4.3)

Since $C_{i-1}\left(b_{1}, b_{2}\right)=\emptyset$ and $i \geq 7$, it follows from (4.1) that $\left|L\left(b_{1}\right)\right|=\left|R\left(b_{2}\right)\right|=3$. Therefore, since $G$ is cubic, there are at least four consecutive out-vertices in $C_{i-1}\left(b_{1}^{\prime}, b_{2}^{\prime}\right)$. Since $C_{i-2}\left(c_{1}, c_{2}\right)=\emptyset$ and since $i-1 \geq 6$, it follows from (4.1) that $\left|L\left(c_{1}\right)\right|=\left|R\left(c_{2}\right)\right|=3$. See Figure 9. Hence $\left|R\left(b_{1}^{\prime}\right)\right|=\left|B\left(b_{1}\right)\right|=\left|B\left(b_{2}\right)\right| \geq 10$.

We claim that $\left|B\left(b_{1}\right)\right|=\left|B\left(b_{2}\right)\right| \geq 12$. For otherwise, $b_{1}^{\prime}$ is adjacent to both $L\left(b_{1}\right)$ and $L\left(c_{1}\right)$, or $b_{2}^{\prime}$ is adjacent to both $R\left(b_{2}\right)$ and $R\left(c_{2}\right)$. By symmetry, we may assume the former. Then since $G$ is cubic, $\left|L\left(b_{1}^{\prime}\right)\right| \geq 5 \leq\left|A\left(b_{1}^{\prime}\right)\right|$. Since $\left|R\left(b_{1}^{\prime}\right)\right| \geq 10, \ell\left(b_{1}^{\prime}\right) \geq$ $(5,5,10)$, a contradiction.

Then $\left|B\left(c_{1}\right)\right|=\left|B\left(c_{2}\right)\right|<12$; for otherwise, $\ell\left(c_{1}\right) \geq(3,12,12)$, a contradiction.

Since $i-2 \geq 5$, it follows from (4.1) that $\left|L\left(d_{1}\right)\right|=\left|R\left(d_{2}\right)\right|=3$ or $\left|C_{i-3}\left(d_{1}, d_{2}\right)\right| \neq 0$. So by (4.2) that $\left|L\left(d_{1}\right)\right|=\left|R\left(d_{2}\right)\right|=3$ or $\left|C_{i-3}\left(d_{1}, d_{2}\right)\right| \geq 2$. Thus $B\left(c_{1}\right)|=| B\left(c_{2}\right) \mid \geq$ 
10. Since $\left|B\left(d_{1}\right)\right| \geq 5 \leq\left|B\left(d_{2}\right)\right|,\left|L\left(d_{1}\right)\right| \leq 4 \geq\left|R\left(d_{2}\right)\right|$ (or else, $\ell\left(d_{1}\right) \geq(5,5,10)$ or $\left.\ell\left(d_{2}\right) \geq(5,5,10)\right)$. Since $\left|B\left(c_{1}\right)\right|=\left|B\left(c_{2}\right)\right|<12$, either $c_{1}^{\prime}$ is adjacent to $L\left(c_{1}\right)$, or $c_{2}^{\prime}$ is adjacent to $R\left(c_{2}\right)$. By symmetry, assume the former. Then $\left|L\left(c_{1}^{\prime}\right)\right|=\left|L\left(d_{1}\right)\right|=4$, and so, $\left|A\left(c_{1}^{\prime}\right)\right| \geq 6$. Since $\left|R\left(c_{1}^{\prime}\right)\right| \geq 10,\left|A\left(c_{1}^{\prime}\right)\right|=6$ (or else $\ell\left(c_{1}^{\prime}\right) \geq(4,7,10)$, a contradiction). So $G$ has an edge $x y$ such that $x \in V\left(C_{i-2}\right), y$ is strictly between $C_{i-1}$ and $C_{i-2}$, and $y$ is adjacent to $L\left(c_{1}\right)$. See Figure 9 . Since $G$ is cubic, we can check that $\ell(y) \geq(4,6,12)$, a contradiction.

(4.4) Lemma. Let $i \geq 8$, let $a_{1}$ and $a_{2}$ be consecutive in-vertices on $C_{i}$ such that (i) $R\left(a_{1}\right)=L\left(a_{2}\right)$ and (ii) $\left|C_{i}\left(a_{1}, a_{2}\right)\right| \geq 4$. Let $b_{1}$ and $b_{2}$ be out-vertices on $C_{i-1}$ such that $R\left(b_{1}\right)=L\left(b_{2}\right)=R\left(a_{1}\right)$. Let $b_{3}, b_{4}$ be the vertices on $C_{i-1}\left(b_{1}, b_{2}\right)$ such that $C_{i-1}\left(b_{1}, b_{3}\right)=\emptyset=C_{i-1}\left(b_{4}, b_{2}\right)$. Assume $\left|C_{i-1}\left(b_{1}, b_{2}\right)\right| \geq 3$. Then

(1) both $b_{3}$ and $b_{4}$ are out-vertices on $C_{i-1}$,

(2) $\left|L\left(b_{1}\right)\right|=\left|R\left(b_{2}\right)\right|=3$ and $\left|R\left(b_{3}\right)\right|=\left|L\left(b_{4}\right)\right|=3$, and

(3) both $R\left(b_{3}\right)$ and $L\left(b_{4}\right)$ use three consecutive vertices on $C_{i-1}$.

Proof. Since $\left|C_{i-1}\left(b_{1}, b_{2}\right)\right| \geq 3,\left|R\left(b_{1}\right)\right|=\left|L\left(b_{2}\right)\right| \geq 10$.

(1) Suppose $b_{3}$ is an in-vertex on $C_{i-1}$. Then $\left|A\left(b_{3}\right)\right|=\left|R\left(b_{1}\right)\right| \geq 11,\left|L\left(b_{3}\right)\right| \geq 5$, and $\left|R\left(b_{3}\right)\right| \geq 4$. Hence, $\left|R\left(b_{3}\right)\right|=4$; otherwise, $\ell\left(b_{3}\right) \geq(5,5,11)$, a contradiction. So let $b, c, c_{3}$ be the vertices of $R\left(b_{3}\right)$ such that $b \in C_{i-1}, c, c_{3} \in C_{i-2}, c$ is adjacent to $b$, and $c_{3}$ is adjacent to $b_{3}$. See Figure 10 .

Since $\left|B\left(b_{1}\right)\right| \geq 5,\left|L\left(b_{1}\right)\right| \leq 4$, for otherwise, $\ell\left(b_{1}\right) \geq(5,5,11)$. In fact $\left|L\left(b_{1}\right)\right|=4$, for otherwise $\left|A\left(b_{3}\right)\right|=\left|R\left(b_{1}\right)\right| \geq 12$ and $\left|L\left(b_{3}\right)\right|=\left|B\left(b_{1}\right)\right| \geq 6$, and so, $\ell\left(b_{3}\right) \geq(4,6,12)$, a contradiction. By (3.3), we see that $\left|L\left(b_{1}\right) \cap C_{i-1}\right| \in\{2,3\}$. Hence we distinguish two cases.

First, assume that $\left|L\left(b_{1}\right) \cap C_{i-1}\right|=2$. See Figure $10(\mathrm{a})$. Then $\left|B\left(b_{1}\right)\right| \geq 6$. Hence $\left|R\left(b_{1}\right)\right|=11$ and $\left|B\left(b_{1}\right)\right|=6$, for otherwise, $\ell\left(b_{1}\right) \geq(4,6,12)$ or $(4,7,11)$. Then $\left|C_{i-1}\left(b_{1}, b_{2}\right)\right|=3$, and $b_{4}$ is also an in-vertex. Hence, $\left|R\left(b_{4}\right)\right| \geq 5,\left|L\left(b_{4}\right)\right| \geq 4$, and $\left|A\left(b_{4}\right)\right|=11$. So $\left|L\left(b_{4}\right)\right|=4$, as otherwise, $\ell\left(b_{4}\right) \geq(5,5,11)$. Thus $b_{4}$ is adjacent to a vertex $c_{4}$ on $C_{i-2}$. Now $\left|B\left(b_{2}\right)\right| \geq 5$. Since $\left|L\left(b_{2}\right)\right|=11, a_{2}$ is adjacent to $b_{2}$, and hence $\left|R\left(b_{2}\right)\right| \geq 4$. Moreover, $\left|R\left(b_{2}\right)\right|=4$ and $\left|B\left(b_{2}\right)\right|=6$, for otherwise, $\ell\left(b_{2}\right) \geq(5,5,11)$ or $(4,7,11)$. Let $c_{1}, c_{2}$ be the out-vertices on $C_{i-2}$ such that $R\left(c_{1}\right)=B\left(b_{1}\right)$ and $L\left(c_{2}\right)=B\left(b_{2}\right)$. Then $c_{1} \neq c_{2}$ (since $i \geq 7$ and $G$ is 2-connected). See Figure 10(a). Since $\left|B\left(b_{1}\right)\right|=\left|B\left(b_{2}\right)\right|=6, C_{i-2}\left(c_{1}, c_{3}\right)=\emptyset=C_{i-2}\left(c_{4}, c_{2}\right)$. Since $i-2 \geq 5$, by (4.1) and (4.2), $\left|B\left(c_{1}\right)\right|=\left|B\left(c_{2}\right)\right| \geq 11$. So $\left|L\left(c_{1}\right)\right| \leq 4$, as otherwise $\ell\left(c_{1}\right) \geq(5,6,10)$. Since $\left|R\left(c_{1}\right)\right|=6$, we have $\left|L\left(c_{1}\right)\right|=4$ and $\left|L\left(c_{1}\right) \cap C_{i-2}\right|=2$. Therefore, $\left|B\left(c_{1}\right)\right| \geq 12$, and so, $\ell\left(c_{1}\right) \geq(4,6,12)$, a contradiction.

So $\left|L\left(b_{1}\right) \cap C_{i-1}\right|=3$. See Figure 10(b) and Figure 10(c). Then $\left|R\left(b_{1}\right)\right| \geq 12$ (since $\left|C_{i-1}\left(b_{1}, b_{2}\right)\right| \geq 3$, and $b_{3}$ and $b$ are in-vertices on $\left.C_{i-1}\right)$. So $\left|B\left(b_{1}\right)\right|=5$, for otherwise, 
$\ell\left(b_{3}\right) \geq(4,6,12)$. Let $c_{1}$ denote the out-vertex on $C_{i-2}$ such that $R\left(c_{1}\right)=B\left(b_{1}\right)$. Then $C_{i-2}\left(c_{1}, c_{3}\right)=\emptyset$, and so, $\left|B\left(c_{1}\right)\right|=|B(c)| \geq 7$. Note that $\left|L\left(c_{1}\right)\right| \in\{5,6\}$, or else $\ell\left(c_{1}\right) \geq(5,7,7)$. Let $d_{1}, d_{2}$ be the out-vertices on $C_{i-3}$ such that $R\left(d_{1}\right)=L\left(d_{2}\right)=B\left(c_{1}\right)$. Assume $\left|L\left(c_{1}\right)\right|=6$. See Figure 10(b). Then $\left|B\left(c_{1}\right)\right|=\left|R\left(d_{1}\right)\right|=7$, for otherwise, $\ell\left(c_{1}\right) \geq$ $(5,6,8)$. Thus $C_{i-3}\left(d_{1}, d_{2}\right)=\emptyset$, and so, $\left|B\left(d_{1}\right)\right| \geq 6$. So $\left|L\left(d_{1}\right)\right|=5$ as otherwise, $\ell\left(d_{1}\right) \geq$ $(6,6,7)$. Since $\left|L\left(d_{1}\right)\right|=5, d_{2} \notin L\left(d_{1}\right)$. Therefore, $\left|B\left(d_{1}\right)\right| \geq 7$, and $\ell\left(d_{1}\right) \geq(5,7,7)$, a contradiction. Now assume $\left|L\left(c_{1}\right)\right|=5$. See Figure 10(c). Then $c \notin L\left(c_{1}\right)$. Let $c_{1}^{\prime}, c_{2}^{\prime}$ denote the in-vertices on $C_{i-2}$ such that $R\left(c_{1}^{\prime}\right)=L\left(c_{2}^{\prime}\right)=B\left(c_{1}\right)$. Then $\left|C_{i-2}\left(c_{1}^{\prime}, c_{2}^{\prime}\right)\right| \geq 4$. Since $i-2 \geq 5$, by (4.1) and (4.2), we have $\left|B\left(c_{1}\right)\right| \geq 10$. Thus $\ell\left(c_{1}\right) \geq(5,5,10)$, a contradiction.

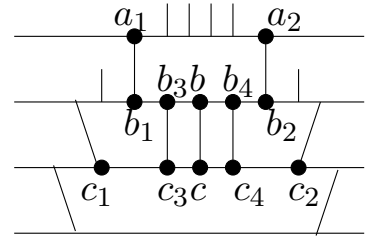

(a)

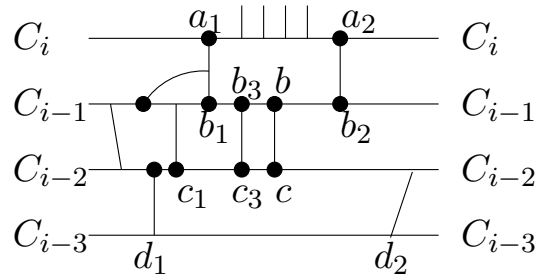

(b)

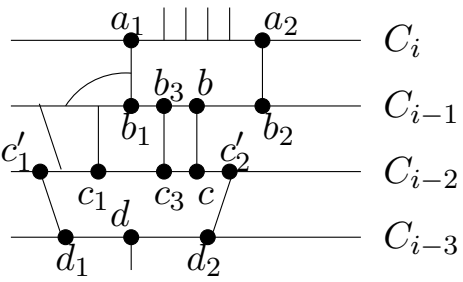

(c)

Figure 10: Proof of Lemma (4.4)

(2) By symmetry, we only prove (2) for $b_{3}$ and $b_{1}$. By (1), $b_{3}$ is an out-vertex, and so, $\left|L\left(b_{3}\right)\right|=\left|R\left(b_{1}\right)\right| \geq 10$ and $\left|B\left(b_{1}\right)\right|=\left|B\left(b_{3}\right)\right| \geq 6$. So $\left|L\left(b_{1}\right)\right| \leq 4$; otherwise, $\ell\left(b_{1}\right) \geq(5,6,10)$, a contradiction.

First, assume that $\left|L\left(b_{1}\right)\right|=4$. Then $\left|L\left(b_{1}\right) \cap C_{i-1}\right| \in\{2,3\}$. If $\left|L\left(b_{1}\right) \cap C_{i-1}\right|=2$, then $\left|B\left(b_{1}\right)\right| \geq 7$, and so, $\ell\left(b_{1}\right) \geq(4,7,10)$, a contradiction. So $\left|L\left(b_{1}\right) \cap C_{i-1}\right|=3$. Then $\left|R\left(b_{1}\right)\right| \geq 11$. Further, $\left|R\left(b_{1}\right)\right|=11$ and $\left|B\left(b_{1}\right)\right|=6$; otherwise, $\ell\left(b_{1}\right) \geq(4,6,12)$ or $\ell\left(b_{1}\right) \geq(4,7,11)$, a contradiction. Because $\left|R\left(b_{1}\right)\right|=11, b_{3}$ is adjacent to $b_{4}$ and $a_{2}$ is adjacent to $b_{2}$. See Figure 11(a). So $\left|B\left(b_{2}\right)\right| \geq 6$ and $\left|R\left(b_{2}\right)\right| \geq 4$, and if $\left|R\left(b_{2}\right)\right|=4$ then $\left|B\left(b_{2}\right)\right| \geq 7$. Thus $\ell\left(b_{2}\right) \geq(4,7,11)$ or $(5,6,11)$, a contradiction.

Thus $\left|L\left(b_{1}\right)\right|=3$. So $\left|B\left(b_{1}\right)\right|=\left|B\left(b_{3}\right)\right| \geq 7$ and $\left|L\left(b_{3}\right)\right|=\left|R\left(b_{1}\right)\right| \geq 11$. Therefore, $\left|R\left(b_{3}\right)\right|=3$, as otherwise $\ell\left(b_{3}\right) \geq(4,7,11)$.

(3) By symmetry, we will only show that $R\left(b_{3}\right)$ uses three consecutive vertices on $C_{i-1}$. Suppose on the contrary that $R\left(b_{3}\right)$ has a vertex, say $b$, not on $C_{i-1}$. See Figure 11(b). Let $b^{\prime}$ denote the vertex in $R\left(b_{3}\right)-\left\{b, b_{3}\right\}$. Note that $C_{i-1}\left(b_{3}, b^{\prime}\right)=\emptyset$ and $\left|R\left(b_{1}\right)\right| \geq 13$ (by (2)). Let $b_{1}^{\prime}, b^{*}$ denote the in-vertices on $C_{i-1}$ such that $R\left(b_{1}^{\prime}\right)=L\left(b^{*}\right)=B\left(b_{1}\right)$. Note that $\left|C_{i-1}\left(b_{1}^{\prime}, b^{*}\right)\right| \geq 4$. Then $b^{*} \in C_{i-1}\left(b^{\prime}, b_{2}\right)$, for otherwise, $\left|B\left(b_{1}\right)\right| \geq 12$ (by (4.1)) and $\ell\left(b_{1}\right) \geq(3,12,13)$. Since $b_{4}$ is an out-vertex and $\left|L\left(b_{4}\right)\right|=3, b$ is not adjacent to $b_{4}$, and so, $\left|R\left(b_{1}\right)\right|=\left|L\left(b_{2}\right)\right| \geq 14$. 


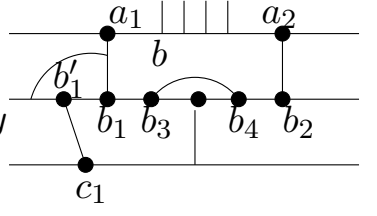

(a)

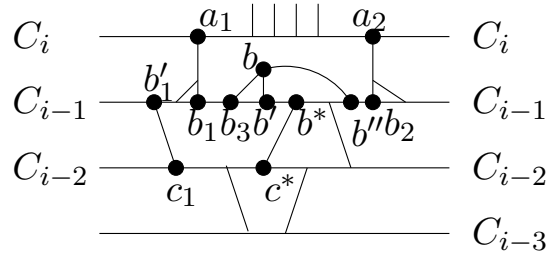

(b)

Figure 11: Proof of Lemma (4.4)

Let $c_{1}, c^{*}$ denote the in-vertices on $C_{i-2}$ such that $R\left(c_{1}\right)=L\left(c^{*}\right)=B\left(b_{1}\right)$.

We claim that $\left|L\left(c_{1}\right)\right|=\left|R\left(c^{*}\right)\right|=3$. Since $\left|C_{i-1}\left(b_{1}^{\prime}, b^{*}\right)\right| \geq 4$ and $i-1 \geq 7$, it follows from (4.1) that $\left|L\left(c_{1}\right)\right|=\left|R\left(c^{*}\right)\right|=3$ or $\left|C_{i-2}\left(c_{1}, c^{*}\right)\right| \neq 0$. If $\left|C_{i-2}\left(c_{1}, c^{*}\right)\right| \neq 0$, then it follows from (4.2) that $\left|C_{i-2}\left(c_{1}, c^{*}\right)\right| \geq 3$. Hence $\left|L\left(c_{1}\right)\right|=\left|R\left(c^{*}\right)\right|=3$.

Thus $\left|B\left(b_{1}\right)\right| \geq 10$ and $\left|L\left(b_{1}^{\prime}\right)\right| \geq 5$. Then $\left|A\left(b_{1}^{\prime}\right)\right| \leq 4$, for otherwise, $\ell\left(b_{1}^{\prime}\right) \geq(5,5,10)$. Hence $b_{1}^{\prime}$ can not be adjacent to $L\left(b_{1}\right)$. So $\left|B\left(b_{1}\right)\right| \geq 11$. Therefore $\ell\left(b_{1}\right) \geq(3,11,14)$, a contradiction.

Let us state the claim in the proof of (3) of (4.4) as a lemma.

(4.5) Lemma. Let $i \geq 8$, let $a_{1}$ and $a_{2}$ be consecutive in-vertices on $C_{i}$ such that (i) $R\left(a_{1}\right)=L\left(a_{2}\right)$ and (ii) $\left|C_{i}\left(a_{1}, a_{2}\right)\right| \geq 4$. Let $b_{1}$ and $b_{2}$ be the out-vertices on $C_{i-1}$ such that $R\left(b_{1}\right)=L\left(b_{2}\right)=R\left(a_{1}\right)$. Then $\left|L\left(b_{1}\right)\right|=\left|R\left(b_{2}\right)\right|=3$.

(4.6) Lemma. Let $i \geq 8$, let $a_{1}$ and $a_{2}$ be consecutive in-vertices on $C_{i}$ such that (i) $R\left(a_{1}\right)=L\left(a_{2}\right)$ and (ii) $\left|C_{i}\left(a_{1}, a_{2}\right)\right| \geq 4$. Let $b_{1}$ and $b_{2}$ be out-vertices on $C_{i-1}$ such that $R\left(b_{1}\right)=L\left(b_{2}\right)=R\left(a_{1}\right)$. Suppose $\left|C_{i-1}\left(b_{1}, b_{2}\right)\right| \geq 3$. Let $b_{3}, b_{4}$ be vertices on $C_{i-1}\left(b_{1}, b_{2}\right)$ such that $C_{i-1}\left(b_{1}, b_{3}\right)=\emptyset=C_{i-1}\left(b_{4}, b_{2}\right)$. Let $b, b^{*}$ be the vertices in $C_{i-1}\left(b_{3}, b_{4}\right)$ such that $C_{i-1}\left(b_{3}, b\right)=\emptyset=C_{i-1}\left(b^{*}, b_{4}\right)$, and let $b^{\prime}, b^{\prime \prime}$ be the neighbors of $b, b^{*}$, respectively, such that $b^{\prime}, b^{\prime \prime} \notin C_{i-1}$. Then

(1) $b^{\prime}, b^{\prime \prime} \notin C_{i-2}$ and

(2) $b^{\prime}$ is contained in a facial triangle of $G$ which also contains two consecutive vertices on $C_{i-2}$, and $b^{\prime \prime}$ is contained in a facial triangle of $G$ which also contains two consecutive vertices on $C_{i-2}$.

Proof. By (4.4), $R\left(b_{3}\right)=A(b)$ and $L\left(b_{4}\right)=A\left(b^{*}\right)$ are facial triangles, and $b$ and $b^{*}$ are in-vertices on $C_{i-1}$. By symmetry, we only need to show the conclusions for $b^{\prime}$.

(1) Suppose $b^{\prime} \in C_{i-2}$. See Figure 12(a). Then $b^{\prime}$ is an out-vertex on $C_{i-2}$, and so, $\left|B\left(b^{\prime}\right)\right| \geq 5$. By (2) of (4.4), $\left|R\left(b^{\prime}\right)\right| \geq 5$ and $\left|L\left(b^{\prime}\right)\right|=\left|B\left(b_{1}\right)\right| \geq 7$. If $\left|B\left(b^{\prime}\right)\right|=5$ then 
$\left|L\left(b^{\prime}\right)\right| \geq 8$ and $\left|R\left(b^{\prime}\right)\right| \geq 6$, and we would have $\ell\left(b^{\prime}\right) \geq(5,6,8)$. So $\left|B\left(b^{\prime}\right)\right| \geq 6$. Then $\left|R\left(b^{\prime}\right)\right|=5$ or else $\ell\left(b^{\prime}\right) \geq(6,6,7)$. So $R\left(b^{\prime}\right) \neq B\left(b_{1}\right)$. Therefore, if $\left|B\left(b^{\prime}\right)\right|=6$ then $\left|L\left(b^{\prime}\right)\right| \geq 8$. So $\ell\left(b^{\prime}\right) \geq(5,6,8)$ or $(5,7,7)$, a contradiction.

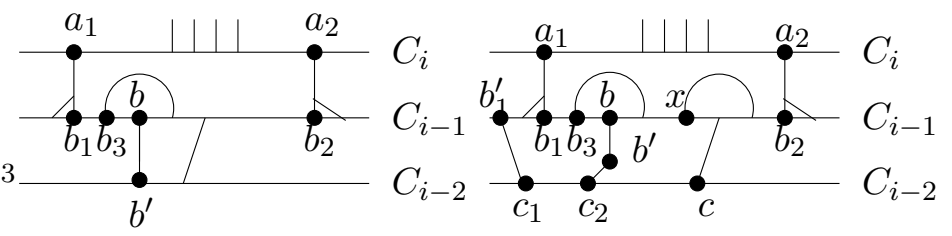

(a)

(b)

Figure 12: (1) of Lemma (4.6)

(2) First we show that $b^{\prime}$ is contained in a facial triangle. Suppose on the contrary that $b^{\prime}$ is not contained in any facial triangle. Since $b^{\prime} \notin C_{i-2},|L(b)| \geq 8$. Hence $|R(b)| \leq 7$ as otherwise $\ell\left(b^{\prime}\right) \geq(4,8,8)$. Thus $b \neq b^{*}$, for otherwise, $|R(b)| \geq 8$. See Figure $12(\mathrm{~b})$. Hence $\left|R\left(b_{1}\right)\right| \geq 14$.

Let $x$ denote the vertex on $C_{i-1}\left(b, b^{*}\right)-A(b)$ such that $x$ is adjacent to $A(b)$. Then $x$ is an out-vertex; otherwise $|L(x)| \geq 6$ and $|R(x)| \geq 4$, and so, $\ell(x) \geq(4,6,14)$, a contradiction. Thus $|R(b)|=7$. This implies that $R(x)$ is a triangle (otherwise $\ell(x) \geq$ $(4,7,14))$ and $R(x)$ uses three consecutive vertices of $C_{i-1}$. Now let $c$ denote the outvertex on $C_{i-2}$ such that $L(c)=R(b)$. Then $|L(c)|=7$. Hence $|R(c)| \geq 5$ and $|B(c)| \geq 6$. Furthermore, if $|R(c)|=5$ then $|B(c)| \geq 7$. So $\ell(c) \geq(5,7,7)$ or $(6,6,7)$, a contradiction.

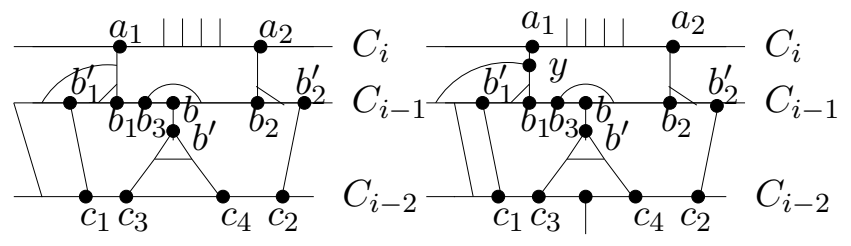

(a)

(b)

Figure 13: (2) of Lemma (4.6)

Next we show that the facial triangle containing $b^{\prime}$ also contains two consecutive vertices on $C_{i-2}$. Note that $\left|R\left(b_{1}\right)\right|=\left|L\left(b_{2}\right)\right| \geq 12$ and $\left|B\left(b_{1}\right)\right| \geq 8 \leq\left|B\left(b_{2}\right)\right|$ (because $b^{\prime}, b^{\prime \prime} \notin C_{i-2}$ by $\left.(1)\right)$. Also note that $\left|B\left(b_{1}\right)\right| \leq 11 \geq\left|B\left(b_{2}\right)\right|$, as otherwise, we would have $\ell\left(b_{1}\right) \geq(3,12,12)$ or $\ell\left(b_{2}\right) \geq(3,12,12)$.

Let $b_{1}^{\prime}, b_{2}^{\prime}$ denote the in-vertices on $C_{i-1}$ such that $R\left(b_{1}^{\prime}\right)=B\left(b_{1}\right)$ and $L\left(b_{2}^{\prime}\right)=B\left(b_{2}\right)$. Let $c_{1}, c_{2}, c_{3}, c_{4}$ be the out-vertices on $C_{i-2}$ such that $R\left(c_{1}\right)=L\left(c_{3}\right)=B\left(b_{1}\right)$ and $L\left(c_{2}\right)=$ 
$R\left(c_{4}\right)=B\left(b_{2}\right)$. See Figure 13 .

For convenience, let $P$ denote the clockwise subpath of $B\left(b_{1}\right)$ from $b^{\prime}$ to $c_{3}$. We wish to show that $|P|=2$, and therefore, since $G$ is cubic, the facial triangle of $G$ containing $b^{\prime}$ also contains two consecutive vertices on $C_{i-2}$.

We claim that $|P| \leq 3$. Suppose this is false, then $|P| \geq 4$. Thus $\left|B\left(b_{1}\right)\right| \geq 10$. Note that $\left|B\left(b_{1}\right)\right| \leq 11$, as otherwise, $\ell\left(b_{1}\right) \geq(3,12,12)$. First, assume that $C_{i-2}\left(c_{1}, c_{3}\right) \neq \emptyset$. Then $\left|B\left(b_{1}\right)\right|=11$, and $C_{i-2}\left(c_{1}, c_{3}\right)$ consists of only one vertex, say $c$. It is easy to see that $\ell(c) \geq(5,5,11)$, a contradiction. So $C_{i-2}\left(c_{1}, c_{3}\right)=\emptyset$. Thus $\left|B\left(c_{1}\right)\right| \geq 6$. So $\left|L\left(c_{1}\right)\right| \leq 4$; otherwise, $\ell\left(c_{1}\right) \geq(5,6,10)$, a contradiction. If $\left|L\left(c_{1}\right)\right|=4$ and $\left|L\left(c_{1}\right) \cap C_{i-2}\right|=2$, then $\left|B\left(c_{1}\right)\right| \geq 7$, and we would have $\ell\left(c_{1}\right) \geq(4,7,10)$. So $\left|L\left(c_{1}\right)\right|=3$ or $\left|L\left(c_{1}\right)\right|=4$ and $\left|L\left(c_{1}\right) \cap C_{i-2}\right|=3$. Then $c_{1}$ is not adjacent to $b_{1}^{\prime}$. So $\left|B\left(b_{1}\right)\right|=11$, and hence, $b_{1}^{\prime}$ is adjacent to $L\left(b_{1}\right)$. Therefore $\left|A\left(b_{1}^{\prime}\right)\right| \geq 5 \leq\left|L\left(b_{1}^{\prime}\right)\right|$, and so, $\ell\left(b_{1}^{\prime}\right) \geq(5,5,11)$, a contradiction.

Now assume that $|P|=3$. Since $b^{\prime}$ is contained in a facial triangle and $|P|=3$, $\left|R\left(c_{3}\right)\right| \geq 4$. See Figure 13 . Note that $9 \leq\left|B\left(b_{1}\right)\right| \leq 11$. Also note that $c_{1} \neq c_{4}$, and so, $c_{3} \notin L\left(c_{1}\right)$.

It is easy to see that $b_{1}^{\prime}$ is adjacent to $L\left(b_{1}\right)$. Otherwise, $\left|C_{i-1}\left(b_{1}^{\prime}, b\right)\right| \geq 4$. By (4.5), $\left|R\left(c_{3}\right)\right|=3$, a contradiction. Thus $\left|A\left(b_{1}^{\prime}\right)\right| \geq 5$.

Assume $\left|A\left(b_{1}^{\prime}\right)\right|=5$. See Figure $13(\mathrm{a})$. Then $\left|L\left(b_{1}^{\prime}\right)\right|=5$ and $\left|R\left(b_{1}^{\prime}\right)\right|=\left|B\left(b_{1}\right)\right|=9$, for otherwise, $\ell\left(b_{1}^{\prime}\right) \geq(5,6,9)$ or $(5,5,10)$. Hence $C_{i-2}\left(c_{1}, c_{3}\right)=\emptyset$ and $\left|B\left(c_{1}\right)\right| \geq 7$. Therefore, $\ell\left(c_{1}\right) \geq(5,7,9)$, a contradiction.

So $\left|A\left(b_{1}^{\prime}\right)\right| \geq 6$. See Figure $13(\mathrm{~b})$. Then $\left|L\left(b_{1}^{\prime}\right)\right|=4$, or else $\ell\left(b_{1}^{\prime}\right) \geq(5,6,9)$. Thus $b_{1}^{\prime}$ is adjacent to $c_{1}$ and $\left|L\left(b_{1}^{\prime}\right) \cap C_{i-2}\right|=2$. Assume $C_{i-2}\left(c_{1}, c_{3}\right)=\emptyset$. Then $\left|B\left(c_{1}\right)\right| \geq 7$. In fact, $\left|B\left(c_{1}\right)\right|=7$, for otherwise, $\ell\left(c_{1}\right) \geq(4,8,9)$. So $\left|R\left(c_{3}\right)\right| \geq 5$, and $\ell\left(c_{3}\right) \geq(5,7,9)$, a contradiction. Thus $C_{i-2}\left(c_{1}, c_{3}\right) \neq \emptyset$, and so, $\left|B\left(b_{1}\right)\right| \geq 10$. This implies that $\left|A\left(b_{1}^{\prime}\right)\right|=6$, or else $\ell\left(b_{1}^{\prime}\right) \geq(4,7,10)$. Thus $A\left(b_{1}^{\prime}\right)$ has an edge $x y$ such that $x \in C_{i-1}, y \in R\left(a_{1}\right) \cap A\left(b_{1}^{\prime}\right)$, and $x, y \notin L\left(b_{1}\right)$. Now it is easy to see that $\ell(y) \geq(4,6,12)$, a contradiction.

(4.7) Lemma. Let $i \geq 10$, let $a_{1}$ and $a_{2}$ be consecutive in-vertices on $C_{i}$ such that (i) $R\left(a_{1}\right)=L\left(a_{2}\right)$ and (ii) $\left|C_{i}\left(a_{1}, a_{2}\right)\right| \geq 4$. Let $b_{1}$ and $b_{2}$ be the out-vertices on $C_{i-1}$ such that $R\left(b_{1}\right)=L\left(b_{2}\right)=R\left(a_{1}\right)$. Suppose $\left|C_{i-1}\left(b_{1}, b_{2}\right)\right| \geq 3$. Then $\left|C_{i-1}\left(b_{1}, b_{2}\right)\right|=3$.

Proof. Let $b_{3}, b_{4}$ be vertices on $C_{i-1}\left(b_{1}, b_{2}\right)$ such that $C_{i-1}\left(b_{1}, b_{3}\right)=\emptyset=C_{i-1}\left(b_{4}, b_{2}\right)$. Let $b, b^{*}$ be the vertices in $C_{i-1}\left(b_{3}, b_{4}\right)$ such that $C_{i-1}\left(b_{3}, b\right)=\emptyset=C_{i-1}\left(b^{*}, b_{4}\right)$.

By (4.4), $|A(b)|=\left|A\left(b^{*}\right)\right|=3=\left|L\left(b_{1}\right)\right|=\left|R\left(b_{2}\right)\right|$, and $A(b)$ and $A\left(b^{*}\right)$ each consist of three consecutive vertices on $C_{i-1}$. So if $b=b^{*}$ then $\left|C_{i-1}\left(b_{1}, b_{2}\right)\right|=3$. Hence we may assume that $b \neq b^{*}$.

Let $b^{\prime}, b^{\prime \prime}$ be the neighbors of $b, b^{*}$, respectively, such that $b^{\prime}, b^{\prime \prime} \notin C_{i-1}$. By (4.6), $b^{\prime}, b^{\prime \prime} \notin C_{i-2}$, there is a facial triangle containing $b^{\prime}$ and two consecutive vertices on $C_{i-2}$ and there is a facial triangle containing $b^{\prime \prime}$ and two consecutive vertices on $C_{i-2}$. 
Since $G$ is cubic, $A(b) \cap A\left(b^{*}\right)=\emptyset$. So $\left|R\left(b_{1}\right)\right|=\left|L\left(b_{2}\right)\right| \geq 14$. Also $\left|B\left(b_{1}\right)\right| \geq 8 \leq$ $\left|B\left(b_{2}\right)\right|$ (since $b^{\prime}, b^{\prime \prime} \notin C_{i-2}$ ), and $\left|B\left(b_{1}\right)\right| \leq 10 \geq\left|B\left(b_{2}\right)\right|$ (otherwise, $\ell\left(b_{1}\right) \geq(3,11,14$ ) or $\ell\left(b_{2}\right) \geq(3,11,14)$, a contradiction).

Let $b_{1}^{\prime}, b_{2}^{\prime}$ be the in-vertex of $C_{i-1}$ such that $R\left(b_{1}^{\prime}\right)=B\left(b_{1}\right)$ and $L\left(b_{2}^{\prime}\right)=B\left(b_{2}\right)$, and let $c_{1}, c_{2}, c_{3}, c_{4}$ be the out-vertices of $C_{i-2}$ such that $R\left(c_{1}\right)=L\left(c_{3}\right)=B\left(b_{1}\right)$ and $R\left(c_{4}\right)=L\left(c_{2}\right)=B\left(b_{2}\right)$. See Figure 14(a). Then by (4.6), $c_{3}$ is adjacent to $b^{\prime}, c_{4}$ is adjacent to $b^{\prime \prime}$, and $\left|R\left(c_{3}\right)\right|=\left|L\left(c_{4}\right)\right|=3$. Since $\left|B\left(b_{1}\right)\right| \leq 10 \geq\left|B\left(b_{2}\right)\right|,\left|C_{i-2}\left(c_{1}, c_{3}\right)\right| \leq$ $2 \geq\left|C_{i-2}\left(c_{4}, c_{2}\right)\right|$.

Case 1. $b_{1}^{\prime}$ is adjacent to $L\left(b_{1}\right)$ or $b_{2}^{\prime}$ is adjacent to $R\left(b_{2}\right)$.

By symmetry, assume that $b_{1}^{\prime}$ is adjacent to $L\left(b_{1}\right)$. Thus $\left|A\left(b_{1}^{\prime}\right)\right| \geq 5$. See Figure 14(b).

We claim that $\left|B\left(b_{1}\right)\right| \leq 9$. Suppose otherwise, then $\left|B\left(b_{1}\right)\right|=10$. So $\left|R\left(b_{1}\right)\right|=14$, as otherwise $\ell\left(b_{1}\right) \geq(3,10,15)$. Then $a_{1}$ is adjacent to $L\left(b_{1}\right)$, and so, $\left|A\left(b_{1}^{\prime}\right)\right| \geq 6$. Then $\left|L\left(b_{1}^{\prime}\right)\right|=4$ as otherwise $\ell\left(b_{1}^{\prime}\right) \geq(5,6,10)$. But then $\left|A\left(b_{1}^{\prime}\right)\right| \geq 7$, and so $\ell\left(b_{1}^{\prime}\right) \geq(4,7,10)$, a contradiction.

Since $\left|B\left(b_{1}\right)\right| \leq 9,\left|C_{i-2}\left(c_{1}, c_{3}\right)\right| \leq 1$. Suppose $\left|C_{i-2}\left(c_{1}, c_{3}\right)\right|=1$. Let $c$ denote the only vertex in $C_{i-2}\left(c_{1}, c_{3}\right)$. Then $c$ is an in-vertex, $|A(c)|=\left|B\left(b_{1}\right)\right|=9$ and $|L(c)| \geq$ 5. Since $\left|R\left(c_{3}\right)\right|=3,|R(c)| \geq 6$. Thus $\ell(c) \geq(5,6,9)$, a contradiction. Therefore $\left|C_{i-2}\left(c_{1}, c_{3}\right)\right|=0$, and hence $\left|B\left(c_{1}\right)\right| \geq 7$. So $\left|L\left(c_{1}\right)\right| \leq 4$, as otherwise, $\ell\left(c_{1}\right) \geq(5,7,8)$.

If $\left|L\left(c_{1}\right)\right|=4$ and $\left|L\left(c_{1}\right) \cap C_{i-2}\right|=2$, then $\left|B\left(c_{1}\right)\right| \geq 8$ and we would have $\ell\left(c_{1}\right) \geq$ $(4,8,8)$. So $\left|L\left(c_{1}\right)\right|=3$ or $\left|L\left(c_{1}\right)\right|=4$ and $\left|L\left(c_{1}\right) \cap C_{i-2}\right|=3$. Then $\left|R\left(b_{1}^{\prime}\right)\right|=\left|R\left(c_{1}\right)\right|=9$ and $\left|L\left(b_{1}^{\prime}\right)\right| \geq 5$. Further, if $\left|L\left(b_{1}^{\prime}\right)\right|=5$, then $\left|A\left(b_{1}^{\prime}\right)\right| \geq 6$. Thus $\ell\left(b_{1}^{\prime}\right) \geq(5,6,9)$, a contradiction.

Case 2. $b_{1}^{\prime}$ is not adjacent to $L\left(b_{1}\right)$ and $b_{2}^{\prime}$ is not adjacent to $R\left(b_{2}\right)$.

Then $\left|C_{i-1}\left(b_{1}^{\prime}, b\right)\right| \geq 4 \leq\left|C_{i-1}\left(b^{*}, b_{2}^{\prime}\right)\right|$. Thus since $i-1 \geq 9$ and by (4.5), $\left|L\left(c_{1}\right)\right|=$ $\left|R\left(c_{2}\right)\right|=3$. Hence $\left|B\left(b_{1}\right)\right| \geq 10 \leq\left|B\left(b_{2}\right)\right|$. In fact, $\left|B\left(b_{1}\right)\right|=10=\left|B\left(b_{2}\right)\right|$ and $\left|R\left(b_{1}\right)\right|=14=\left|L\left(b_{2}\right)\right|$; for otherwise $\ell\left(b_{1}\right) \geq(3,11,14)$ or $(3,10,15)$. Thus there are exactly six vertices in $C_{i-1}\left(b_{1}, b_{2}\right)$, and $C_{i-2}\left(c_{1}, c_{3}\right)=\emptyset=C_{i-2}\left(c_{4}, c_{2}\right)$.

Let $c_{1}^{\prime}$ and $c^{\prime}$ denote the in-vertices on $C_{i-2}$ such that $R\left(c_{1}^{\prime}\right)=L\left(c^{\prime}\right)=B\left(c_{1}\right)$. We claim that $c^{\prime} \in C_{i-2}\left(c_{3}, c_{4}\right)$. Otherwise, $\left|C_{i-2}\left(c, c^{\prime}\right)\right| \geq 8$. See Figure $14(\mathrm{c})$. Since $i-2 \geq 8$ and by (4.5), $R\left(c_{1}^{\prime}\right) \geq 14$. Thus $R\left(c_{1}^{\prime}\right)=14=\left|B\left(c_{1}\right)\right|$, or else $\ell\left(c_{1}\right) \geq(3,10,15)$. Then $c_{1}^{\prime}$ is adjacent to $L\left(c_{1}\right)$ and $\left|A\left(c_{1}^{\prime}\right)\right| \geq 6$. Therefore $\ell\left(c_{1}^{\prime}\right) \geq(4,6,14)$, a contradiction.

If $c^{\prime}$ is adjacent to $R\left(c_{3}\right)$ or $L\left(c_{4}\right)$, then it is easy to check that $\ell\left(c^{\prime}\right) \geq(4,8,8)$, a contradiction.

So $c^{\prime}$ is not adjacent to $R\left(c_{3}\right)$ and $c^{\prime}$ is not adjacent to $L\left(c_{4}\right)$. Let $x$ be the vertex in $C_{i-2}\left(c_{3}, c_{4}\right)-V\left(R\left(c_{3}\right) \cup L\left(c_{4}\right)\right)$ such that $x$ is adjacent to $R\left(c_{3}\right)$. See Figure $14(\mathrm{~d})$. Then $x$ is an out-vertex on $C_{i-2}$ and $|L(x)|=|R(b)| \geq 10$. Further, $|L(x)|=|R(b)|=10$, as otherwise, $\ell(b) \geq(3,11,14)$. Also $|R(x)|=|L(y)|=3$, or else $\ell(x) \geq(4,10,10)$. Hence $\left|C_{i-2}\left(c_{3}, c_{4}\right)\right|=5=\left|C_{i-2}\left(c_{1}^{\prime}, c^{\prime}\right)\right|$. Let $y$ be the vertex in $C_{i-2}\left(c^{\prime}, c_{4}\right)$ and let $c_{2}^{\prime}$ 


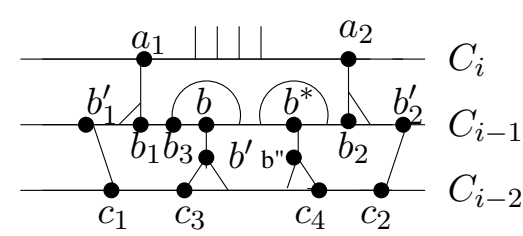

(a)

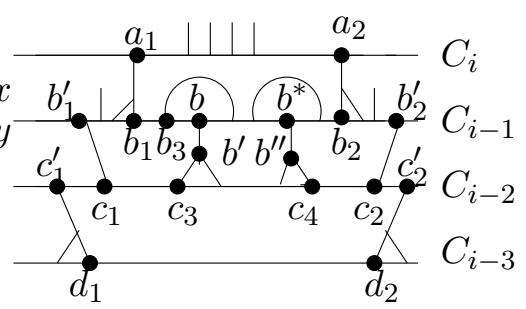

(c)

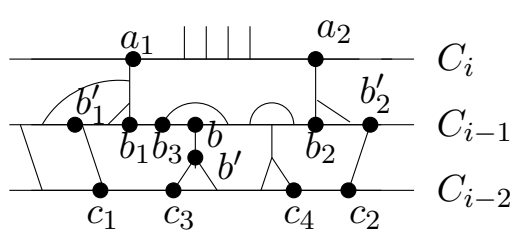

(b)

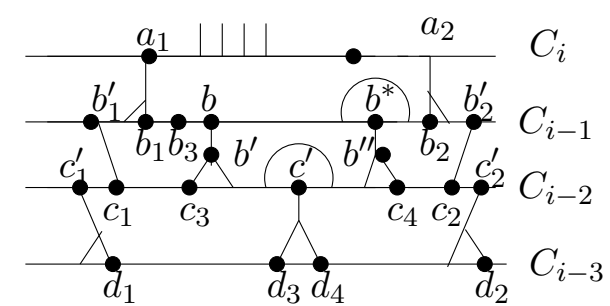

(d)

Figure 14: Proof of Lemma (4.7)

be the in-vertex on $C_{i-2}$ such that $L\left(c_{2}^{\prime}\right)=B\left(c_{2}\right)$. Let $d_{1}, d_{2}, d_{3}, d_{4}$ be out-vertices on $C_{i-3}$ such that $R\left(d_{1}\right)=L\left(d_{3}\right)=B\left(c_{1}\right)=B\left(c_{3}\right)$ and $R\left(d_{4}\right)=L\left(d_{2}\right)=B\left(c_{2}\right)=B\left(c_{4}\right)$. Since $C_{i-2}\left(c_{1}, c_{3}\right)=\emptyset$ and $\left|C_{i-1}\left(b_{1}^{\prime}, b\right)\right| \geq 4$, it follows from (4.3) that $C_{i-3}\left(d_{1}, d_{3}\right) \neq \emptyset$. Similarly, we can show that $C_{i-3}\left(d_{4}, d_{2}\right) \neq \emptyset$. Since $i-2 \geq 8$ and by $(4.5),\left|L\left(d_{1}\right)\right|=$ $\left|R\left(d_{3}\right)\right|=3=\left|L\left(d_{4}\right)\right|=\left|R\left(d_{2}\right)\right|$. So $\left|L\left(c^{\prime}\right)\right| \geq 12 \leq\left|R\left(c^{\prime}\right)\right|$. Thus $\ell\left(c^{\prime}\right) \geq(3,12,12)$, a contradiction.

(4.8) Lemma. Let $i \geq 11$, let $a_{1}$ and $a_{2}$ be consecutive in-vertices on $C_{i}$ such that (i) $R\left(a_{1}\right)=L\left(a_{2}\right)$ and (ii) $\left|C_{i}\left(a_{1}, a_{2}\right)\right| \geq 4$. Let $b_{1}, b_{2}$ denote the out-vertices on $C_{i-1}$ such that $R\left(b_{1}\right)=L\left(b_{2}\right)=R\left(a_{1}\right)$. Then $\left|C_{i-1}\left(b_{1}, b_{2}\right)\right|=\emptyset$.

Proof. Suppose on the contrary that $\left|C_{i-1}\left(b_{1}, b_{2}\right)\right| \neq 0$. Since $i \geq 11$ and by (4.2), $\left|C_{i-1}\left(b_{1}, b_{2}\right)\right| \geq 3$. Therefore, by $(4.7),\left|C_{i-1}\left(b_{1}, b_{2}\right)\right|=3$. Let $b_{3}, b, b_{4}$ be the vertices on $C_{i-1}\left(b_{1}, b_{2}\right)$ in this clockwise order from $b_{1}$ to $b_{2}$. See Figure 15 . By $(4.4),\left|L\left(b_{1}\right)\right|=$ $\left|R\left(b_{2}\right)\right|=3$ and $|A(b)|=\left|R\left(b_{3}\right)\right|=\left|L\left(b_{4}\right)\right|=3$. Let $b^{\prime}$ be the neighbor of $b$ not on $C_{i-1}$. By (4.6), $b^{\prime} \notin C_{i-2}$ and there is a facial triangle containing $b^{\prime}$ and two consecutive vertices on $C_{i-2}$. Let $b_{1}^{\prime}, b_{2}^{\prime}$ denote the in-vertices on $C_{i-1}$ such that $R\left(b_{1}^{\prime}\right)=B\left(b_{1}\right)$ and $L\left(b_{2}^{\prime}\right)=$ $B\left(b_{2}\right)$. Let $c_{1}, c_{2}, c_{3}, c_{4}$ denote the out-vertices on $C_{i-2}$ such that $R\left(c_{1}\right)=L\left(c_{3}\right)=B\left(b_{1}\right)$ and $R\left(c_{2}\right)=L\left(c_{4}\right)=B\left(b_{2}\right)$.

Note that $\left|R\left(b_{1}\right)\right|=\left|L\left(b_{2}\right)\right| \geq 12$. So $\left|B\left(b_{1}\right)\right| \leq 11 \geq\left|B\left(b_{2}\right)\right|$, for otherwise we would have $\ell\left(b_{1}\right) \geq(3,12,12)$ or $\ell\left(b_{2}\right) \geq(3,12,12)$. Also note that $\left|B\left(b_{1}\right)\right| \geq 8 \leq\left|B\left(b_{2}\right)\right|$.

Case $1 . b_{1}^{\prime}$ is adjacent to $L\left(b_{1}\right)$, or $b_{2}^{\prime}$ is adjacent to $R\left(b_{2}\right)$. 


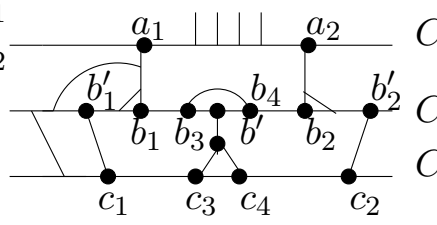

(a)

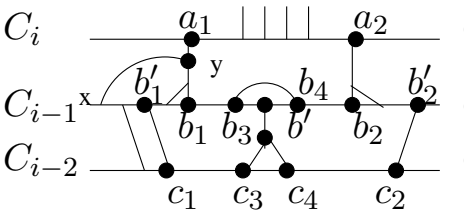

(b)



(c)

Figure 15: Proof of Lemma (4.8)

By symmetry we may assume that $b_{1}^{\prime}$ is adjacent to $L\left(b_{1}\right)$. Then $\left|A\left(b_{1}^{\prime}\right)\right| \geq 5$. First, assume $\left|A\left(b_{1}^{\prime}\right)\right|=5$. See Figure $15(\mathrm{a})$. Then $\left|A\left(b_{1}^{\prime}\right) \cap C_{i-1}\right|=3$ and $\left|L\left(b_{1}^{\prime}\right)\right| \geq 5$. In fact $\left|L\left(b_{1}^{\prime}\right)\right|=5$ and $\left|L\left(b_{1}^{\prime}\right) \cap C_{i-2}\right|=2$, for otherwise $\ell\left(b_{1}^{\prime}\right) \geq(5,6,8)$. So $b_{1}^{\prime}$ is adjacent to $c_{1}$ and $\left|B\left(c_{1}\right)\right| \geq 6$. Hence $\ell\left(c_{1}\right) \geq(5,6,8)$, a contradiction. So $\left|A\left(b_{1}^{\prime}\right)\right| \geq 6$. Then $\left|L\left(b_{1}^{\prime}\right)\right| \leq 4$, as otherwise, we would have $\ell\left(b_{1}^{\prime}\right) \geq(5,6,8)$. In fact $\left|L\left(b_{1}^{\prime}\right)\right|=4$ (since $G$ is cubic), $b_{1}^{\prime}$ is adjacent to $c_{1}$, and $\left|L\left(b_{1}^{\prime}\right) \cap C_{i-2}\right|=2$. See Figure $15(\mathrm{~b})$. Note that $c_{4} \notin L\left(b_{1}^{\prime}\right)$ (because $G$ is cubic and $\left.\left|L\left(b_{1}^{\prime}\right)\right|=4\right)$. Then $C_{i-2}\left(c_{1}, c_{3}\right) \neq \emptyset$, otherwise, $\left|B\left(c_{1}\right)\right| \geq 8$, and we would have $\ell\left(c_{1}\right) \geq(4,8,8)$. Suppose $C_{i-2}\left(c_{1}, c_{3}\right)$ consists of only one vertex, say $c$. Then $|L(c)| \geq 6,|R(c)| \geq 6$, and $|A(c)| \geq 9$. So $\ell(c) \geq(6,6,9)$, a contradiction. Hence, $\left|C_{i-2}\left(c_{1}, c_{3}\right)\right| \geq 2$. Then $\left|B\left(b_{1}\right)\right| \geq 10$. So $\left|A\left(b_{1}^{\prime}\right)\right|=6$, as otherwise, $\ell\left(b_{1}^{\prime}\right) \geq(4,7,10)$. Thus $A\left(b_{1}^{\prime}\right)$ has an edge $x y$ such that $x \in C_{i-1}, y \in R\left(a_{1}\right) \cap A\left(b_{1}^{\prime}\right)$, and $x, y \notin L\left(b_{1}\right)$. Now it is easy to see that $\ell(y) \geq(4,6,12)$, a contradiction.

Case $2 . b_{1}^{\prime}$ is not adjacent to $L\left(b_{1}\right)$, and $b_{2}^{\prime}$ is not adjacent to $R\left(b_{2}\right)$.

Then $\left|C_{i-1}\left(b_{1}^{\prime}, b\right)\right| \geq 4$ and $\left|C_{i-1}\left(b, b_{2}^{\prime}\right)\right| \geq 4$. Since $i-1 \geq 10$ and by $(4.5),\left|L\left(c_{1}\right)\right|=$ $\left|R\left(c_{2}\right)\right|=3$. Thus $\left|B\left(b_{1}\right)\right| \geq 10 \leq\left|B\left(b_{2}\right)\right|,\left|B\left(c_{1}\right)\right| \geq 6 \leq\left|B\left(c_{2}\right)\right|$ and $L\left(c_{1}\right) \cap R\left(c_{2}\right)=\emptyset$.

We claim that $C_{i-2}\left(c_{1}, c_{3}\right)=\emptyset=C_{i-2}\left(c_{4}, c_{2}\right)$. For otherwise, we may assume by symmetry that $C_{i-2}\left(c_{1}, c_{3}\right) \neq \emptyset$. Then $\left|B\left(b_{1}\right)\right| \geq 11$. Thus $\left|B\left(b_{1}\right)\right|=11$ and $C_{i-2}\left(c_{1}, c_{3}\right)$ consists of only one vertex, say $c$. Now $\ell(c) \geq(6,6,11)$, a contradiction.

Let $c_{1}^{\prime}, c_{2}^{\prime}$ denote the in-vertices on $C_{i-2}$ such that $R\left(c_{1}^{\prime}\right)=L\left(c_{2}^{\prime}\right)=B\left(c_{1}\right)$, and let $d_{1}, d_{2}$ denote the out-vertices on $C_{i-3}$ such that $R\left(d_{1}\right)=L\left(d_{2}\right)=B\left(c_{1}\right)$. See Figure $15(\mathrm{c})$.

Since $i-2 \geq 9$ and $\left|C_{i-2}\left(c_{1}^{\prime}, c_{2}^{\prime}\right)\right| \geq 4$, it follows from (4.5) that $\left|L\left(d_{1}\right)\right|=\left|R\left(d_{2}\right)\right|=3$. Thus $\left|B\left(c_{1}\right)\right| \geq 12$. Since $\left|C_{i-1}\left(b_{1}^{\prime}, b\right)\right| \geq 4$ and $C_{i-2}\left(c_{1}, c_{3}\right)=\emptyset$, it follows from (4.3) that $\left|C_{i-3}\left(d_{1}, d_{2}\right)\right| \neq 0$. By $(4.2),\left|C_{i-3}\left(d_{1}, d_{2}\right)\right| \geq 3$. Therefore, $\left|B\left(c_{1}\right)\right| \geq 14$. In fact $\left|B\left(c_{1}\right)\right|=14$, as otherwise, $\ell\left(c_{1}\right) \geq(3,10,15)$. So $c_{1}^{\prime}$ is adjacent to $L\left(c_{1}\right)$. Then $\left|A\left(c_{1}^{\prime}\right)\right| \geq 5$. Since $\left|L\left(d_{1}\right)\right|=3, \ell\left(c_{1}^{\prime}\right) \geq(5,5,14)$, a contradiction.

We are now ready to prove our main lemma in this section. 
(4.9) Lemma. Let $i \geq 12$, let $a_{1}$ and $a_{2}$ be consecutive in-vertices on $C_{i}$ such that $R\left(a_{1}\right)=L\left(a_{2}\right)$. Then $\left|C_{i}\left(a_{1}, a_{2}\right)\right| \leq 3$.

Proof. Suppose for a contradiction that $\left|C_{i}\left(a_{1}, a_{2}\right)\right| \geq 4$. Then by $(4.5),\left|C_{i-1}\left(b_{1}, b_{2}\right)\right|=0$. So by (4.1), $\left|L\left(b_{1}\right)\right|=\left|R\left(b_{2}\right)\right|=3$. Let $b_{1}^{\prime}, b_{2}^{\prime}$ be the in-vertices on $C_{i-1}$ such that $R\left(b_{1}^{\prime}\right)=$ $L\left(b_{2}^{\prime}\right)=B\left(b_{1}\right)$, and let $c_{1}, c_{2}$ be the out-vertices on $C_{i-2}$ such that $R\left(c_{1}\right)=L\left(c_{2}\right)=B\left(b_{1}\right)$. See Figure 16.

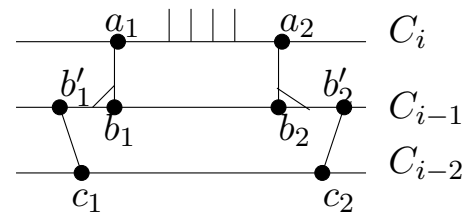

Figure 16: Proof of Lemma (4.9)

Then $\left|C_{i-1}\left(b_{1}^{\prime}, b_{2}^{\prime}\right)\right| \geq 4$. Since $i-1 \geq 11$ and by $(4.3),\left|C_{i-2}\left(c_{1}, c_{2}\right)\right| \neq 0$. Since $i-1 \geq 11$, we have a contradiction to (4.8).

\section{Three vertices between consecutive in-vertices}

In this section we show that for sufficiently large $i$, there are at most two vertices of $C_{i}$ between any two consecutive in-vertices of $C_{i}$. As in Section 4, this is done through a series of lemmas.

(5.1) Lemma. Let $i \geq 15$, let $a_{1}$ and $a_{2}$ be consecutive in-vertices on $C_{i}$ such that (i) $R\left(a_{1}\right)=L\left(a_{2}\right)$ and (ii) $\left|C_{i}\left(a_{1}, a_{2}\right)\right|=3$. Let $b_{1}, b_{2}$ denote the out-vertices on $C_{i-1}$ such that $R\left(b_{1}\right)=L\left(b_{2}\right)=R\left(a_{1}\right)$. Then $\left|C_{i-1}\left(b_{1}, b_{2}\right)\right| \geq 2$.

Proof. Suppose on the contrary that $\left|C_{i-1}\left(b_{1}, b_{2}\right)\right| \leq 1$. Let $b_{1}^{\prime}, b_{2}^{\prime}$ denote the in-vertices on $C_{i-1}$ such that $R\left(b_{1}^{\prime}\right)=B\left(b_{1}\right)$ and $B\left(b_{2}\right)=L\left(b_{2}^{\prime}\right)$. Let $c_{1}, c_{2}$ be the out-vertices on $C_{i-2}$ such that $R\left(c_{1}\right)=R\left(b_{1}^{\prime}\right)$ and $L\left(c_{2}\right)=L\left(b_{2}^{\prime}\right)$. See Figure 17 .

Case 1. $\left|C_{i-1}\left(b_{1}, b_{2}\right)\right|=1$.

Let $b$ be the only vertex in $C_{i-1}\left(b_{1}, b_{2}\right)$. See Figure $17($ a). Since $G$ is 2-connected, $b$ is an in-vertex. Note that $|A(b)| \geq 8$ and $|L(b)| \geq 5 \leq|R(b)|$. In fact, $|L(b)|=|R(b)|=5$; for otherwise, $\ell(b) \geq(5,6,8)$. So $C_{i-1}\left(b_{1}^{\prime}, b_{1}\right)=\emptyset=C_{i-1}\left(b_{2}, b_{2}^{\prime}\right)$. Hence $c_{1} \neq c_{2}, b_{1}^{\prime} \neq b_{2}^{\prime}$, $\left|B\left(c_{1}\right)\right| \geq 7$, and $\left|C_{i-2}\left(c_{1}, c_{2}\right)\right|=1$.

Moreover, $a_{1}$ is adjacent to $b_{1}$, or $a_{2}$ is adjacent to $b_{2}$. Otherwise, $\left|R\left(b_{1}\right)\right| \geq 10$, and $\ell(b) \geq(5,5,10)$. By symmetry assume that $a_{1}$ is adjacent to $b_{1}$. Then $\left|L\left(b_{1}\right)\right| \geq 5$. 


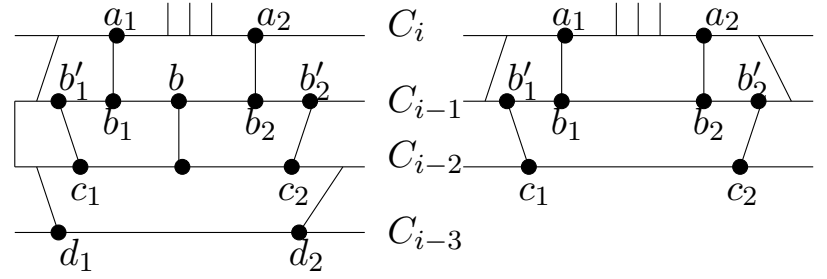

(a) (b)

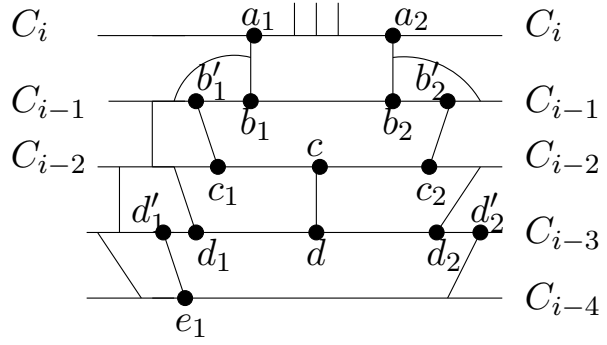

(c)

Figure 17: Proof of Lemma (5.1)

Further, $\left|L\left(b_{1}\right)\right|=5$, for otherwise $\ell\left(b_{1}\right) \geq(5,6,8)$. So $\left|L\left(b_{1}\right) \cap C_{i-1}\right|=3$. This implies that $\left|L\left(c_{1}\right)\right| \geq 5$.

We claim that $\left|L\left(c_{1}\right)\right|=6$ and $\left|L\left(c_{1}\right) \cap C_{i-2}\right|=3$. If $\left|L\left(c_{1}\right) \cap C_{i-2}\right|=2$, then there are four consecutive out-vertices on $C_{i-2}$, contradicting (4.9). So $\left|L\left(c_{1}\right) \cap C_{i-2}\right| \geq 3$. So $\left|L\left(c_{1}\right)\right|=6$, or else $\ell\left(c_{1}\right) \geq(5,7,7)$. Since $\left|L\left(b_{1}\right) \cap C_{i-1}\right|=3$ and $\left|L\left(c_{1}\right) \cap C_{i-2}\right| \geq 3$, we have $\left|L\left(c_{1}\right) \cap C_{i-2}\right|=3$.

So $\left|B\left(c_{1}\right)\right|=7$, for otherwise $\ell\left(c_{1}\right) \geq(5,6,8)$. Now let $d_{1}, d_{2}$ denote the out-vertices on $C_{i-3}$ such that $R\left(d_{1}\right)=L\left(d_{2}\right)=B\left(c_{1}\right)$. Since $\left|B\left(c_{1}\right)\right|=7=\left|R\left(d_{1}\right)\right|, C_{i-3}\left(d_{1}, d_{2}\right)=\emptyset$. So $\left|B\left(d_{1}\right)\right| \geq 6$ and $\left|L\left(d_{1}\right)\right| \geq 5$. Since $\left|L\left(c_{1}\right) \cap C_{i-2}\right|=3,\left|L\left(d_{1}\right)\right|=5$ implies $\left|B\left(d_{1}\right)\right| \geq 7$. So $\ell\left(d_{1}\right) \geq(5,7,7)$ or $(6,6,7)$, a contradiction.

Case 2. $\left|C_{i-1}\left(b_{1}, b_{2}\right)\right|=0$.

By $(4.9), C_{i-1}\left(b_{1}^{\prime}, b_{1}\right)=\emptyset$ or $C_{i-1}\left(b_{2}, b_{2}^{\prime}\right)=\emptyset$. By symmetry, we may assume $C_{i-1}\left(b_{1}^{\prime}, b_{1}\right)=\emptyset$. So $\left|L\left(b_{1}\right)\right| \geq 4,\left|R\left(b_{1}\right)\right| \geq 7$, and $\left|B\left(b_{1}\right)\right| \geq 6$. See Figure $17(\mathrm{~b})$.

Claim 1. $\left|L\left(b_{1}\right)\right|=4$ and $\left|L\left(b_{1}\right) \cap C_{i-1}\right|=3$.

Suppose Claim 1 is false. Then $\left|L\left(b_{1}\right)\right| \geq 5$. In fact $\left|L\left(b_{1}\right)\right|=5$ as otherwise $\ell\left(b_{1}\right) \geq$ $(6,6,7)$. Moreover, $a_{2}$ is adjacent to $b_{2}$ and $a_{1}$ is adjacent to $b_{1}$, for otherwise $\ell\left(b_{1}\right) \geq$ $(5,6,8)$. So $\left|L\left(b_{1}\right) \cap C_{i-1}\right| \geq 3$. See Figure $17(\mathrm{~b})$.

Also $\left|B\left(b_{1}\right)\right|=6$, or else $\ell\left(b_{1}\right) \geq(5,7,7)$. So $C_{i-2}\left(c_{1}, c_{2}\right)=\emptyset, c_{1}$ is adjacent to $b_{1}^{\prime}, c_{2}$ is adjacent to $b_{2}^{\prime}$, and $C_{i-1}\left(b_{2}, b_{2}^{\prime}\right)=\emptyset$. Thus $\left|R\left(c_{1}\right)\right|=6$ and $\left|B\left(c_{1}\right)\right| \geq 6$.

Hence $\left|L\left(c_{1}\right)\right|=5$, as otherwise $\ell\left(c_{1}\right) \geq(6,6,6)$. Therefore $\left|L\left(c_{1}\right) \cap C_{i-2}\right|=2$. Since $C_{i-1}\left(b_{2}, b_{2}^{\prime}\right)=\emptyset$ and because $a_{2}$ is adjacent to $b_{2},\left|R\left(b_{2}\right)\right| \geq 5$. So by a symmetric argument as above, we have $\left|R\left(c_{2}\right)\right| \geq 5$ and $\left|R\left(c_{2}\right) \cap C_{i-2}\right|=2$. Then $C_{i-2}$ has four distinct consecutive out-vertices, contradicting (4.9).

So $\left|L\left(b_{1}\right)\right|=4$. Therefore, since $\left|C_{i-1}\left(b_{1}^{\prime}, b_{1}\right)\right|=0,\left|L\left(b_{1}\right) \cap C_{i-1}\right|=3$.

Claim 2. $\left|R\left(b_{2}\right)\right|=4$ and $\left|R\left(b_{2}\right) \cap C_{i-1}\right|=3$. 
Since $\left|L\left(b_{1}\right)\right|=4$ and $\left|L\left(b_{1}\right) \cap C_{i-1}\right|=3,\left|R\left(b_{1}\right)\right| \geq 8$ and $\left|L\left(c_{1}\right)\right| \geq 5$. See Figure 17(b) (but with $\left|L\left(b_{1}\right)\right|=4$ and $\left|L\left(b_{1}\right) \cap C_{i-1}\right|=3$ ).

Assume $C_{i-1}\left(b_{2}, b_{2}^{\prime}\right) \neq \emptyset$. Then $\left|B\left(b_{1}\right)\right| \geq 7$. In fact, $\left|B\left(b_{1}\right)\right|=7$, for otherwise $\ell\left(b_{1}\right) \geq(4,8,8)$. So $C_{i-2}\left(c_{1}, c_{2}\right)=\emptyset$ and $c_{1}$ is adjacent to $b_{1}^{\prime}$. Therefore, $\left|B\left(c_{1}\right)\right| \geq 6$, $\left|L\left(c_{1}\right)\right| \geq 5$, and $\left|R\left(c_{1}\right)\right|=\left|B\left(b_{1}\right)\right|=7$. Moreover, if $\left|L\left(c_{1}\right)\right|=5$ then $\left|B\left(c_{1}\right)\right| \geq 7$. Hence $\ell\left(c_{1}\right) \geq(5,7,7)$ or $(6,6,7)$, a contradiction.

Thus $C_{i-1}\left(b_{2}, b_{2}^{\prime}\right)=\emptyset$. Therefore $\left|R\left(b_{2}\right)\right| \geq 4$. In fact $\left|R\left(b_{2}\right)\right|=4$, otherwise $\ell\left(b_{2}\right) \geq$ $(5,6,8)$. So $\left|R\left(b_{2}\right) \cap C_{i-1}\right|=3$.

By Claims 1 and $2,\left|R\left(b_{1}\right)\right|=\left|L\left(b_{2}\right)\right| \geq 9,\left|L\left(c_{1}\right)\right| \geq 5$, and $\left|R\left(c_{2}\right)\right| \geq 5$. See Figure $17(\mathrm{c})$.

Claim 3. $\left|C_{i-2}\left(c_{1}, c_{2}\right)\right|=1$.

Suppose $\left|C_{i-2}\left(c_{1}, c_{2}\right)\right|=0$. Then $\left|B\left(c_{2}\right)\right|=\left|B\left(c_{1}\right)\right| \geq 6$. Hence $\left|R\left(c_{2}\right)\right|=\left|L\left(c_{1}\right)\right|=5$, for otherwise $\ell\left(c_{1}\right) \geq(6,6,6)$ or $\ell\left(c_{2}\right) \geq(6,6,6)$. So $\left|L\left(c_{1}\right) \cap C_{i-2}\right|=2=\mid R\left(c_{2}\right) \cap$ $C_{i-2} \mid$. Then there are four consecutive out-vertices on $C_{i-2}$, contradicting (4.9). So $C_{i-2}\left(c_{1}, c_{2}\right) \neq \emptyset$. In fact $\left|C_{i-2}\left(c_{1}, c_{2}\right)\right|=1$, as otherwise $\left|B\left(b_{1}\right)\right| \geq 8$ and $\ell\left(b_{1}\right) \geq(4,8,9)$.

By Claim 3, let $c$ denote the only vertex in $C_{i-2}\left(c_{1}, c_{2}\right)$. Then $|L(c)| \geq 5 \leq|R(c)|$. By the symmetry between $R(c)$ and $L(c)$, we may assume that $|L(c)|=5$, otherwise, $\ell(c) \geq(6,6,7)$. Then $\left|L\left(c_{1}\right)\right| \geq 6$. In fact $\left|L\left(c_{1}\right)\right|=6$, or else $\ell\left(c_{1}\right) \geq(5,7,7)$. Similarly, $|R(c)| \in\{5,6\}$, for otherwise $\ell(c) \geq(5,7,7)$. Let $d, d_{1}, d_{2}$ denote the out-vertices on $C_{i-3}$ such that $d$ is adjacent to $c, R\left(d_{1}\right)=B\left(c_{1}\right)$, and $L\left(d_{2}\right)=B\left(c_{2}\right)$. Because $|L(c)|=5$, $C_{i-3}\left(d_{1}, d\right)=\emptyset$.

We claim that $C_{i-3}\left(d, d_{2}\right)=\emptyset$. For otherwise, $\left|B\left(c_{2}\right)\right|=|R(c)|=6$. So $\left|R\left(c_{2}\right)\right| \geq 6$, and $\ell\left(c_{2}\right) \geq(6,6,7)$, a contradiction.

Hence $d_{1}, d, d_{2}$ are consecutive out-vertices on $C_{i-3}$. Let $d_{1}^{\prime}, d_{2}^{\prime}$ denote the in-vertices on $C_{i-3}$ such that $R\left(d_{1}^{\prime}\right)=B\left(d_{1}\right)=B\left(d_{2}\right)=L\left(d_{2}^{\prime}\right)$. Let $e_{1}$ be the out-vertex on $C_{i-4}$ such that $R\left(e_{1}\right)=B\left(d_{1}\right)$. Then, since $i-3 \geq 12$ and by (4.9), $C_{i-3}\left(d_{1}, d_{2}\right)=\emptyset$. So $\left|B\left(d_{1}\right)\right|=\left|R\left(d_{1}^{\prime}\right)\right| \geq 7$ and $\left|A\left(d_{1}^{\prime}\right)\right| \geq 6$. Hence $\left|L\left(d_{1}^{\prime}\right)\right|=5$, as otherwise $\ell\left(d_{1}^{\prime}\right) \geq(6,6,7)$. Therefore $d_{1}^{\prime}$ is adjacent to $e_{1},\left|L\left(e_{1}\right)\right| \geq 5,\left|B\left(e_{1}\right)\right| \geq 6$. Moreover, if $\left|L\left(e_{1}\right)\right|=5$ then either $\left|B\left(e_{1}\right)\right| \geq 7$ or $\left|R\left(e_{1}\right)\right| \geq 8$. So $\ell\left(e_{1}\right) \geq(5,7,7)$ or $(5,6,8)$, a contradiction.

(5.2) Lemma. Let $i \geq 15$, let $a_{1}$ and $a_{2}$ be consecutive in-vertices on $C_{i}$ such that (i) $R\left(a_{1}\right)=L\left(a_{2}\right)$ and (ii) $\left|C_{i}\left(a_{1}, a_{2}\right)\right|=3$. Let $b_{1}, b_{2}$ denote the out-vertices on $C_{i-1}$ such that $R\left(b_{1}\right)=L\left(b_{2}\right)=R\left(a_{1}\right)$. Suppose $\left|C_{i}\left(a_{1}, a_{2}\right)\right|=3$. Then $\left|C_{i-1}\left(b_{1}, b_{2}\right)\right| \geq 3$.

Proof. Suppose on the contrary $\left|C_{i-1}\left(b_{1}, b_{2}\right)\right| \leq 2$. By (5.1), $C_{i-1}\left(b_{1}, b_{2}\right)$ has exactly two vertices, say $b_{3}$ and $b_{4}$. See Figure 18. Without loss of generality, assume that $b_{3} \in C_{i-1}\left(b_{1}, b_{4}\right)$. Since $G$ is 2-connected and by $(3.4)$, both $b_{3}$ and $b_{4}$ are in-vertices. So $\left|R\left(b_{1}\right)\right|=\left|L\left(b_{2}\right)\right| \geq 9$. Note that $\left|L\left(b_{3}\right)\right|=\left|B\left(b_{1}\right)\right| \geq 5 \leq\left|B\left(b_{2}\right)\right|=\left|R\left(b_{4}\right)\right|$. So $\left|R\left(b_{3}\right)\right|=\left|L\left(b_{4}\right)\right| \geq 5$, for otherwise $\ell\left(b_{3}\right) \geq(5,6,9)$. 
Case 1. $\left|R\left(b_{3}\right)\right|=\left|L\left(b_{4}\right)\right|=5$.

Then $\left|B\left(b_{1}\right)\right|=\left|B\left(b_{2}\right)\right|=5$, as otherwise $\ell\left(b_{1}\right) \geq(5,6,9)$ or $\ell\left(b_{2}\right) \geq(5,6,9)$. Therefore $\left|R\left(b_{3}\right) \cap C_{i-2}\right|=3$. Let $c$ be the only vertex in $R\left(b_{3}\right) \cap C_{i-2}$. Then $c$ is an in-vertex on $C_{i-2}$ and $|L(c)| \geq 6 \leq|R(c)|$. See Figure 18(a). Note that $c$ has a neighbor, say $d$, on $C_{i-3}$; for otherwise, $\ell(c) \geq(5,7,7)$. Note that $|L(d)| \geq 6 \leq|R(d)|$ and $|B(d)| \geq 5$. Further, if $|B(d)|=5$ then $|L(d)| \geq 7 \leq|R(d)|$. So $\ell(d) \geq(5,7,7)$ or $(6,6,6)$, a contradiction.

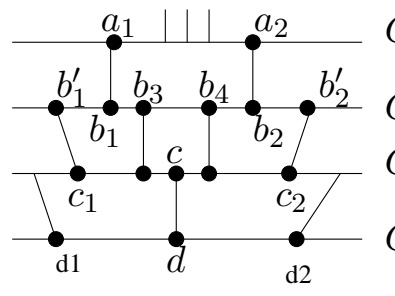

(a)

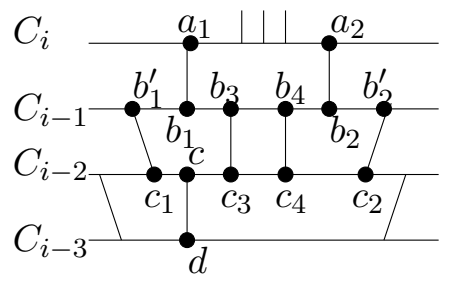

(b)

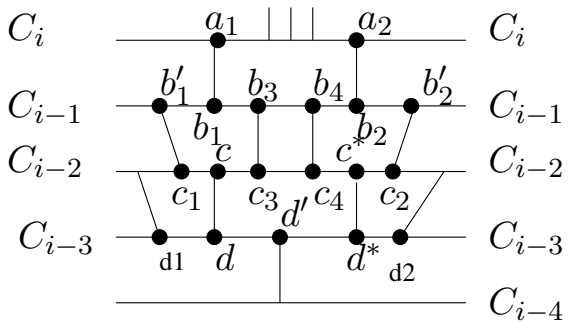

(c)

Figure 18: Proof of Lemma (5.2)

Let $c_{3}, c_{4} \in C_{i-2}$ be the neighbors of $b_{3}, b_{4}$, respectively. Let $c_{1}, c_{2}$ be the out-vertices on $C_{i-2}$ such that $R\left(c_{1}\right)=B\left(b_{1}\right)$ and $L\left(c_{2}\right)=B\left(b_{2}\right)$. See Figure $18(\mathrm{~b})$.

We claim that $\left|C_{i-2}\left(c_{1}, c_{3}\right)\right| \leq 1 \geq\left|C_{i-2}\left(c_{4}, c_{2}\right)\right|$. For otherwise, we may assume by symmetry that $\left|C_{i-2}\left(c_{1}, c_{3}\right)\right| \geq 2$. Then $\left|L\left(b_{3}\right)\right| \geq 7$. In fact, $\left|L\left(b_{3}\right)\right|=7$, or else $\ell\left(b_{3}\right) \geq(4,8,9)$. Therefore $\left|L\left(b_{1}\right)\right| \geq 5$, and $\ell\left(b_{1}\right) \geq(5,7,9)$, a contradiction.

We further claim that $C_{i-2}\left(c_{1}, c_{3}\right) \cup C_{i-2}\left(c_{4}, c_{2}\right)$ has an in-vertex on $C_{i-2}$. For otherwise, $C_{i-2}\left(c_{1}, c_{3}\right) \cup C_{i-2}\left(c_{4}, c_{2}\right)=\emptyset$. Since $G$ is cubic and 2-connected, $c_{1} \neq c_{2}$ and $\left|C_{i-2}\left(c_{2}, c_{1}\right)\right| \neq \emptyset$. Therefore, $C_{i-2}$ has four consecutive out-vertices, contradicting (4.9).

By symmetry we may assume that $C_{i-2}\left(c_{1}, c_{3}\right)$ has an in-vertex. So let $c$ denote the only vertex in $C_{i-2}\left(c_{1}, c_{3}\right)$. See Figure 18(b). Then $|A(c)| \geq 6$ and $|L(c)| \geq 5$. Hence $c$ is adjacent to some vertex $d$ on $C_{i-3}$, for otherwise $\ell(c) \geq(6,6,6)$.

Suppose $C_{i-2}\left(c_{4}, c_{2}\right)=\emptyset$. Then $|R(c)| \geq 7$. So $|L(c)|=5$ and $|R(c)|=7$; otherwise, we would have $\ell(c) \geq(5,6,8)$ or $(6,6,7)$. Then $|L(d)|=5,|R(d)|=7$, and $|B(d)| \geq 7$. This implies $\ell(d) \geq(5,7,7)$, a contradiction.

So $\left|C_{i-2}\left(c_{4}, c_{2}\right)\right|=1$. Let $c^{*}$ denote the only vertex in $C_{i-2}\left(c_{4}, c_{2}\right)$. See Figure 18(c). Then $c^{*}$ is adjacent to some vertex $d^{*}$ in $C_{i-3}$, for otherwise $\ell\left(c^{*}\right) \geq(6,6,6)$. Note that $|R(c)|=\left|L\left(c^{*}\right)\right| \geq 6$. Then $|L(c)|=5=\left|R\left(c^{*}\right)\right|$, otherwise $\ell(c) \geq(6,6,6)$ or $\ell\left(c^{*}\right) \geq(6,6,6)$. So $|R(c)|=\left|L\left(c^{*}\right)\right| \leq 7$, or else $\ell(c) \geq(5,6,8)$. Since $i-3 \geq 12$ and by (4.9), $C_{i-3}\left(d, d^{*}\right) \neq \emptyset$. So $\left|C_{i-3}\left(d, d^{*}\right)\right|=1$ (because $\left.|R(c)| \leq 7\right)$. Let $d^{\prime}$ denote the only vertex in $C_{i-3}\left(d, d^{*}\right)$. Then $\ell\left(d^{\prime}\right) \geq(6,6,7)$, a contradiction. 
(5.3) Lemma. Let $i \geq 17$, let $a_{1}$ and $a_{2}$ be consecutive in-vertices on $C_{i}$ such that (i) $R\left(a_{1}\right)=L\left(a_{2}\right)$ and (ii) $\left|C_{i}\left(a_{1}, a_{2}\right)\right|=3$. Let $b_{1}, b_{2}$ denote the out-vertices on $C_{i-1}$ such that $R\left(b_{1}\right)=L\left(b_{2}\right)=R\left(a_{1}\right)$, and let $b_{3}, b_{4}$ be the vertice in $C_{i-1}\left(b_{1}, b_{2}\right)$ such that $C_{i-1}\left(b_{1}, b_{3}\right)=\emptyset=C_{i-1}\left(b_{4}, b_{2}\right)$. Then both $b_{3}$ and $b_{4}$ are in-vertices.

Proof. Suppose this lemma is false. By symmetry assume that $b_{3}$ is an out-vertex. Then $\left|B\left(b_{1}\right)\right|=\left|B\left(b_{3}\right)\right| \geq 6$. By $(5.2),\left|C_{i-1}\left(b_{1}, b_{2}\right)\right| \geq 3$, and so, $\left|R\left(b_{1}\right)\right|=\left|L\left(b_{2}\right)\right| \geq 9$. Hence $\left|L\left(b_{1}\right)\right| \leq 4$, as otherwise, $\ell\left(b_{1}\right) \geq(5,6,9)$. Let $b_{1}^{\prime}, b$ be the in-vertices on $C_{i-1}$ such that $R\left(b_{1}^{\prime}\right)=L(b)=B\left(b_{1}\right)$, and let $c_{1}, c$ be the out-vertices on $C_{i-2}$ such that $R\left(c_{1}\right)=L(c)=B\left(b_{1}\right)$. See Figure 19. Note that $b \in C_{i-1}\left(b_{3}, b_{4}\right)$ as otherwise $G$ has a 2-cut contained in $V\left(C_{i-1}\right)$, contradicting (3.4). Since $\left|L\left(b_{1}\right)\right| \leq 4$, we have two cases to consider.

Case 1. $\left|L\left(b_{1}\right)\right|=3$.

Then $\left|R\left(b_{1}\right)\right| \geq 10$. Since $i-1 \geq 16$ and $\left|C_{i-1}\left(b_{1}^{\prime}, b\right)\right| \geq 4$, it follows from (5.2) that $\left|C_{i-2}\left(c_{1}, c_{3}\right)\right| \geq 3$, and so, $\left|B\left(b_{1}\right)\right| \geq 9$. Hence $\left|R\left(b_{3}\right)\right|=3$, as otherwise $\ell\left(b_{3}\right) \geq(4,9,10)$. By $(4.9), b_{1}^{\prime}$ is adjacent to $L\left(b_{1}\right)$ and $C_{i-1}\left(b_{3}, b\right)=\emptyset$. As a consequence, $\left|A\left(b_{1}^{\prime}\right)\right| \geq 5$ and $R\left(b_{3}\right) \subset C_{i-1}$. See Figure 19(a).

We claim that $b$ is not adjacent to $c$. For otherwise, $|L(c)| \geq 9$ and $|R(c)| \geq 5 \leq|B(c)|$. Further, if $|B(c)|=5$ then $|R(c)| \geq 6$. Thus $\ell(c) \geq(5,6,9)$, a contradiction.

Hence, $|L(c)|=\left|R\left(b_{1}^{\prime}\right)\right| \geq 10$. Then $\left|L\left(b_{1}^{\prime}\right)\right|=4$, or else $\ell\left(b_{1}^{\prime}\right) \geq(5,5,10)$. Therefore $\left|A\left(b_{1}^{\prime}\right)\right| \geq 6$. In fact, $\left|A\left(b_{1}^{\prime}\right)\right|=6$ as otherwise, $\ell\left(b_{1}^{\prime}\right) \geq(4,7,10)$. So $R\left(a_{1}\right) \cap A\left(b_{1}^{\prime}\right) \neq \emptyset$. Let $y$ be the vertex in $R\left(a_{1}\right) \cap A\left(b_{1}^{\prime}\right)$ such that the clockwise path in $R\left(a_{1}\right)$ from $y$ to $a_{1}$ is shortest. So $\left|R\left(a_{1}\right)\right| \geq 11$. Let $C$ denote the facial cycle of $G$ containing $y$ such that $C \neq R\left(a_{1}\right)$ and $C \neq A\left(b_{1}^{\prime}\right)$. Note that $|C| \geq 4$. If $|C| \geq 5$, then $\ell(y) \geq(5,6,11)$. If $|C|=4$, then $\left|R\left(a_{1}\right)\right| \geq 12$ and $\ell(y) \geq(4,6,12)$.

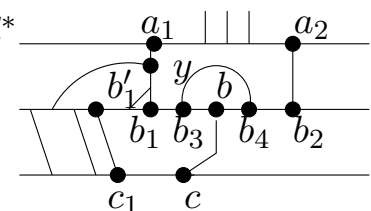

(a)
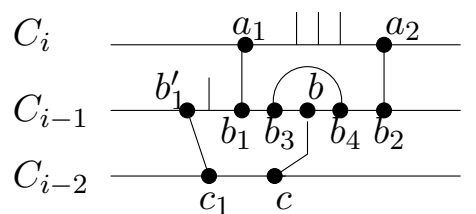

(b)

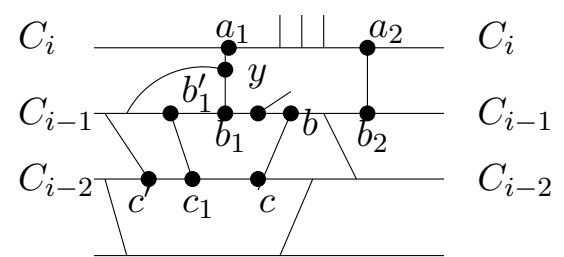

(c)

Figure 19: Proof of Lemma (5.3)

Case 2. $\left|L\left(b_{1}\right)\right|=4$.

First, assume that $\left|L\left(b_{1}\right) \cap C_{i-1}\right|=2$. See Figure 19(b). Then $\left|C_{i-1}\left(b_{1}^{\prime}, b\right)\right| \geq 3$. Since $i-1 \geq 16$ and by $(5.2),\left|B\left(b_{1}\right)\right| \geq 9$. Thus $\ell\left(b_{1}\right) \geq(4,9,9)$, a contradiction. 
So $\left|L\left(b_{1}\right) \cap C_{i-1}\right|=3$. See Figure $19(\mathrm{c})$. Then $\left|R\left(b_{1}\right)\right| \geq 10$. Thus $\left|B\left(b_{1}\right)\right|=6$, as otherwise $\ell\left(b_{1}\right) \geq(4,7,10)$. So $b$ is adjacent to $c, b_{1}^{\prime}$ is adjacent to $c_{1},\left|C_{i-1}\left(b_{1}^{\prime}, b\right)\right|=2$, and $C_{i-2}\left(c_{1}, c\right)=\emptyset$. Now $\left|B\left(c_{1}\right)\right| \geq 6$. Because $\left|L\left(b_{1}\right) \cap C_{i-1}\right|=3$ and $\left|L\left(b_{1}\right)\right|=4$, $\left|L\left(c_{1}\right)\right| \geq 5$. In fact, $\left|L\left(c_{1}\right)\right|=5$ and $\left|L\left(c_{1}\right) \cap C_{i-2}\right|=2$, as otherwise $\ell\left(c_{1}\right) \geq(6,6,6)$. Let $c^{\prime}$ be the vertex on $C_{i-2}$ such that $R\left(c^{\prime}\right)=L\left(c_{1}\right)$. Then $c^{\prime}, c_{1}$ and $c$ are three consecutive out-vertices on $C_{i-2}$. Since $i-2 \geq 15$ and by $(5.1),\left|B\left(c_{1}\right)\right| \geq 9$. Thus $\ell\left(c_{1}\right) \geq(5,6,9)$, a contradiction.

(5.4) Lemma. Let $i \geq 17$, let $a_{1}$ and $a_{2}$ be consecutive in-vertices on $C_{i}$ such that $R\left(a_{1}\right)=L\left(a_{2}\right)$. Then $\left|C_{i}\left(a_{1}, a_{2}\right)\right| \leq 2$.

Proof. Suppose this lemma is false. Then by (4.9), $\left|C_{i}\left(a_{1}, a_{2}\right)\right|=3$. Let $b_{1}, b_{2}$ denote the out-vertices on $C_{i-1}$ such that $R\left(b_{1}\right)=L\left(b_{2}\right)=R\left(a_{1}\right)$. By $(5.2),\left|C_{i-1}\left(b_{1}, b_{2}\right)\right| \geq 3$. Let $b_{3}, b_{4}$ be vertices in $C_{i-1}\left(b_{1}, b_{2}\right)$ such that $C_{i-1}\left(b_{1}, b_{3}\right)=\emptyset=C_{i-1}\left(b_{4}, b_{2}\right)$. By (5.3), both $b_{3}$ and $b_{4}$ are in-vertices. See Figure 20. So $\left|R\left(b_{1}\right)\right|=\left|L\left(b_{2}\right)\right| \geq 10$ and $\left|B\left(b_{1}\right)\right|=$ $\left|L\left(b_{3}\right)\right| \geq 5 \leq\left|R\left(b_{4}\right)\right|=\left|B\left(b_{2}\right)\right|$.

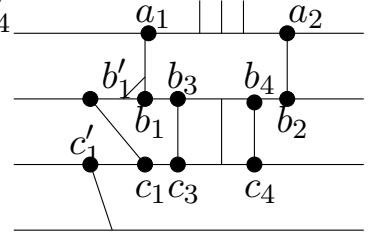

(a)

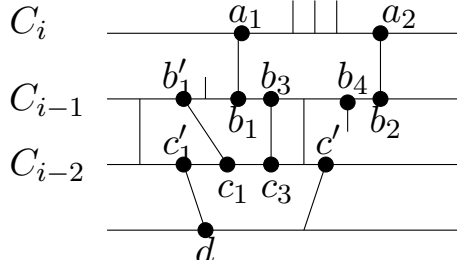

(b)

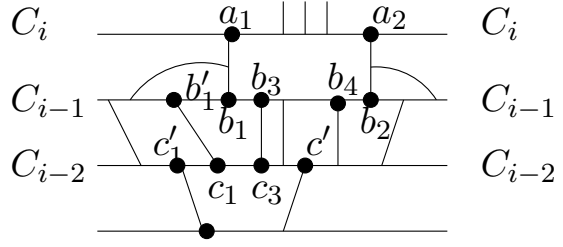

(c)

Figure 20: Proof of Lemma (5.4)

Then $\left|R\left(b_{3}\right)\right|=4=\left|L\left(b_{4}\right)\right|$, for otherwise $\ell\left(b_{3}\right) \geq(5,5,10)$ or $\ell\left(b_{4}\right) \geq(5,5,10)$. Also $\left|L\left(b_{1}\right)\right| \leq 4 \geq\left|R\left(b_{2}\right)\right|$, otherwise, $\ell\left(b_{1}\right) \geq(5,5,10)$ or $\ell\left(b_{2}\right) \geq(5,5,10)$.

Let $b_{1}^{\prime}$ be the in-vertex on $C_{i-1}$ such that $R\left(b_{1}^{\prime}\right)=B\left(b_{1}\right)$. Let $c_{1}, c_{3}, c_{4}$ be out-vertice on $C_{i-2}$ such that $R\left(c_{1}\right)=L\left(c_{3}\right)=B\left(b_{1}\right)$ and $R\left(c_{4}\right)=B\left(b_{2}\right)$. Since $\left|R\left(b_{3}\right)\right|=\left|L\left(b_{4}\right)\right|=4$, $c_{3}$ is adjacent to $b_{3}$ and $c_{4}$ is adjacent to $b_{4}$. Let $c_{1}^{\prime}, c^{*}$ be the in-vertices on $C_{i-2}$ such that $R\left(c_{1}^{\prime}\right)=L\left(c^{*}\right)=B\left(c_{1}\right)$.

We claim that $\left|L\left(b_{1}\right)\right|=4=\left|R\left(b_{2}\right)\right|$. For otherwise, by symmetry we may assume that $\left|L\left(b_{1}\right)\right|=3$. See Figure $20(\mathrm{a})$. Then $\left|R\left(b_{1}\right)\right| \geq 11$ and $\left|B\left(b_{1}\right)\right| \geq 6$. Indeed, $\left|R\left(b_{1}\right)\right|=11$ and $\left|B\left(b_{1}\right)\right|=6$, as otherwise $\ell\left(b_{3}\right) \geq(4,7,11)$ or $(4,6,12)$. So $\left|C_{i-1}\left(b_{1}, b_{2}\right)\right|=3$. Since $\left|R\left(b_{3}\right)\right|=\left|L\left(b_{4}\right)\right|=4, C_{i-2}\left[c_{3}, c_{4}\right]$ consists of three consecutive out-vertices on $C_{i-2}$. Since $i-2 \geq 15$ and by (4.9), $c_{1}$ and $c_{3}$ cannot be adjacent. Thus $\left|B\left(b_{1}\right)\right| \geq 7$, a contradiction.

We further claim that $\left|L\left(b_{1}\right) \cap C_{i-1}\right|=3=\left|R\left(b_{2}\right) \cap C_{i-1}\right|$. For otherwise, by symmetry we may assume $\left|L\left(b_{1}\right) \cap C_{i-1}\right|=2$. See Figure $20(\mathrm{~b})$. Then $\left|B\left(b_{1}\right)\right| \geq 6$. Indeed 
$\left|B\left(b_{1}\right)\right|=6$, otherwise $\ell\left(b_{1}\right) \geq(4,7,10)$. Thus $b_{3}$ is adjacent to $c_{3}, b_{1}^{\prime}$ is adjacent to $c_{1}$, and $C_{i-2}\left(c_{1}, c_{3}\right)=\emptyset$. Thus $\left|C_{i-2}\left(c_{1}^{\prime}, c^{*}\right)\right| \geq 3$ (because $\left|R\left(b_{3}\right)\right|=4$ ). By (4.9), $C_{i-2}\left(c_{1}^{\prime}, c_{1}\right)=\emptyset$, and so $\left|L\left(c_{1}\right)\right| \geq 5$. By $(5.2),\left|B\left(c_{1}\right)\right| \geq 9$. Since $\left|R\left(c_{1}\right)\right|=\left|B\left(b_{1}\right)\right|=6$, $\ell\left(c_{1}\right) \geq(5,6,9)$, a contradiction.

So $\left|R\left(b_{1}\right)\right|=\left|L\left(b_{2}\right)\right| \geq 12$. See Figure $20(\mathrm{c})$. Then $\left|L\left(b_{3}\right)\right|=5$, otherwise $\ell\left(b_{3}\right) \geq$ $(4,6,12)$. Thus $b_{1}^{\prime}$ is adjacent to $c_{1}$, and $C_{i-2}\left(c_{1}, c_{3}\right)=\emptyset$. Again, $\left|C_{i-2}\left(c_{1}^{\prime}, c^{*}\right)\right| \geq 3$ (because $\left.\left|R\left(b_{3}\right)\right|=4\right)$. By $(4.9), C_{i-2}\left(c_{1}^{\prime}, c_{1}\right)=\emptyset$, and so $\left|L\left(c_{1}\right)\right| \geq 6$. By $(5.2),\left|B\left(c_{1}\right)\right| \geq$ 9. Therefore $\ell\left(c_{1}\right) \geq(5,6,9)$, a contradiction.

\section{Two vertices between consecutive in-vertices}

In this section, we show that for sufficiently large $i$, there is at most one vertex between any two consecutive in-vertices on $C_{i}$. Again this is done through a series of lemmas.

(6.1) Lemma. Let $i \geq 20$, let $a_{1}$ and $a_{2}$ be consecutive in-vertices on $C_{i}$ such that (i) $R\left(a_{1}\right)=L\left(a_{2}\right)$ and (ii) $\left|C_{i}\left(a_{1}, a_{2}\right)\right|=2$. Let $b_{1}, b_{2}$ denote the out-vertices on $C_{i-1}$ such that $R\left(b_{1}\right)=L\left(b_{2}\right)=R\left(a_{1}\right)$. Then $\left|C_{i-1}\left(b_{1}, b_{2}\right)\right| \neq 0$.

Proof. Suppose $\left|C_{i-1}\left(b_{1}, b_{2}\right)\right|=0$. Then $\left|B\left(b_{1}\right)\right|=\left|B\left(b_{2}\right)\right| \geq 6$. Let $b_{1}^{\prime}, b_{2}^{\prime}$ be the invertices on $C_{i-1}$ such that $R\left(b_{1}^{\prime}\right)=B\left(b_{1}\right)=L\left(b_{2}^{\prime}\right)$, and let $c_{1}, c_{2}$ denote the out-vertices on $C_{i-2}$ such that $R\left(c_{1}\right)=L\left(c_{2}\right)=B\left(b_{1}\right)$. Because $i-1 \geq 19$ and by $(5.4),\left|C_{i-1}\left(b_{1}^{\prime}, b_{2}^{\prime}\right)\right|=2$.

Case 1. $\left|C_{i-2}\left(c_{1}, c_{2}\right)\right|=0$.

Let $c_{1}^{\prime}, c_{2}^{\prime}$ denote the in-vertices on $C_{i-2}$ such that $R\left(c_{1}^{\prime}\right)=B\left(c_{1}\right)=L\left(c_{2}^{\prime}\right)$. See Figure $21(\mathrm{a})$. Since $i-2 \geq 18$ and by (5.4), $\left|C_{i-2}\left(c_{1}^{\prime}, c_{2}^{\prime}\right)\right|=2$. Thus $C_{i-2}\left(c_{1}^{\prime}, c_{1}\right)=$ $\emptyset=C_{i-2}\left(c_{2}, c_{2}^{\prime}\right)$. Note that $\left|B\left(c_{2}\right)\right| \geq 6$. Then $\left|L\left(c_{1}\right)\right|=\left|R\left(c_{2}\right)\right|=5$, otherwise $\ell\left(c_{1}\right) \geq$ $(6,6,6)$ or $\ell\left(c_{2}\right) \geq(6,6,6)$. This forces $\left|L\left(b_{1}\right)\right| \geq 5 \leq\left|R\left(b_{2}\right)\right|$. Further $\left|L\left(b_{1}\right)\right|=5=$ $\left|R\left(b_{2}\right)\right|$, otherwise $\ell\left(b_{1}\right) \geq(6,6,6)$ or $\ell\left(b_{2}\right) \geq(6,6,6)$. Therefore $\left|R\left(b_{1}\right)\right|=\left|L\left(b_{2}\right)\right| \geq 8$, and so $\ell\left(b_{1}\right) \geq(5,6,8)$, a contradiction.

Case 2. $\left|C_{i-2}\left(c_{1}, c_{2}\right)\right|=1$.

Let $c$ be the only vertex in $C_{i-2}\left(c_{1}, c_{2}\right)$. See Figure $21(\mathrm{~b})$. Then $|A(c)| \geq 7$ and $|L(c)| \geq 5 \leq|R(c)|$. If $\left|L(c) \cap C_{i-3}\right|=2=\left|R(c) \cap C_{i-3}\right|$, then $C_{i-3}$ has three consecutive out-vertices, contradicting (5.4) (because $i-3 \geq 17$ ). So by symmetry assume that $\left|R(c) \cap C_{i-3}\right| \geq 3$. Hence $|R(c)| \geq 6$. Indeed $|R(c)|=6$ and $|L(c)|=5$, otherwise $\ell(c) \geq(5,7,7)$ or $(6,6,7)$. So $\left|R(c) \cap C_{i-2}\right|=3$, and hence, $\left|R\left(c_{2}\right)\right| \geq 5$. In fact, $\left|R\left(c_{2}\right)\right|=5$, for otherwise $\ell\left(c_{2}\right) \geq(6,6,7)$. Therefore $\left|R\left(b_{2}\right)\right| \geq 5$. Further, if $\left|R\left(b_{2}\right)\right|=5$ then $\left|L\left(b_{2}\right)\right| \geq 7$. So $\ell\left(b_{2}\right) \geq(5,7,7)$ or $(6,6,7)$, a contradicton.

Case 3. $\left|C_{i-2}\left(c_{1}, c_{2}\right)\right| \geq 2$. 


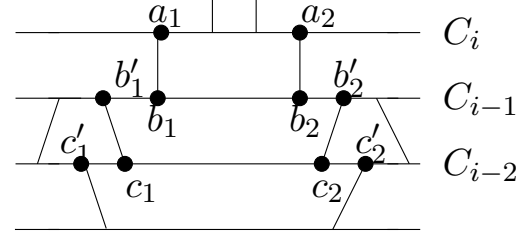

(a)



(b)

Figure 21: Proof of Lemma (6.1)

Then $\left|B\left(b_{1}\right)\right|=\left|B\left(b_{2}\right)\right| \geq 8$. Hence $\left|L\left(b_{1}\right)\right|=\left|R\left(b_{2}\right)\right|=4$; otherwise $\ell\left(b_{1}\right) \geq(5,6,8)$ or $\ell\left(b_{2}\right) \geq(5,6,8)$, a contradiction. Since $i-1 \geq 19$ and by $(5.4),\left|L\left(b_{1}\right) \cap C_{i-1}\right|=3=\mid R\left(b_{2}\right) \cap$ $C_{i-1} \mid$. Thus $\ell\left(b_{1}\right) \geq(4,8,8)$, a contradiction.

(6.2) Lemma. Let $i \geq 21$, let $a_{1}$ and $a_{2}$ be consecutive in-vertices on $C_{i}$ such that (i) $R\left(a_{1}\right)=L\left(a_{2}\right)$ and (ii) $\left|C_{i}\left(a_{1}, a_{2}\right)\right|=2$. Let $b_{1}, b_{2}$ denote the out-vertices on $C_{i-1}$ such that $R\left(b_{1}\right)=L\left(b_{2}\right)=R\left(a_{1}\right)$. Then $\left|C_{i-1}\left(b_{1}, b_{2}\right)\right| \neq 1$.

Proof. Suppose $\left|C_{i-1}\left(b_{1}, b_{2}\right)\right|=1$. Then $\left|R\left(b_{1}\right)\right|=\left|L\left(b_{2}\right)\right| \geq 7$. Let $b$ be the only vertex in $C_{i-1}\left(b_{1}, b_{2}\right)$. Since $G$ is 2 -connected, $b$ is an in-vertex. See Figure 22. Note that $|L(b)|=\left|B\left(b_{1}\right)\right| \geq 5 \leq\left|B\left(b_{2}\right)\right|=|R(b)|$.



Figure 22: Proof of Lemma (6.2)

Now $|L(b)|=5$ or $|R(b)|=5$, as otherwise $\ell(b) \geq(6,6,7)$. By symmetry assume that $|L(b)|=5$. Then $b$ is adjacent to a vertex on $C_{i-3}$, say $c$. Let $c_{1}$ denote the outvertex on $C_{i-2}$ such that $R\left(c_{1}\right)=B\left(b_{1}\right)$, and let $b_{1}^{\prime}$ be the in-vertex on $C_{i-1}$ such that $R\left(b_{1}^{\prime}\right)=B\left(b_{1}\right)$. Since $|L(b)|=5, C_{i-2}\left(c_{1}, c\right)=\emptyset, b_{1}^{\prime}$ is adjacent to $c_{1}$, and $C_{i-1}\left(b_{1}^{\prime}, b_{1}\right)=\emptyset$.

Let $c_{1}^{\prime}, c^{\prime}$ denote the in-vertices on $C_{i-2}$ such that $R\left(c_{1}^{\prime}\right)=L\left(c^{\prime}\right)=B\left(c_{1}\right)$. Since $i-2 \geq 19$ and by (5.4), $\left|C_{i-2}\left(c_{1}^{\prime}, c^{\prime}\right)\right|=2$ and $C_{i-2}\left(c, c^{\prime}\right)=\emptyset$. Thus $\left|A\left(c^{\prime}\right)\right| \geq 6$. Indeed, 
$\left|A\left(c^{\prime}\right)\right|=6$, as otherwise $\ell(b) \geq(5,7,7)$. Now $\left|R\left(c^{\prime}\right)\right|=5$ and $\left|L\left(c^{\prime}\right)\right| \leq 7$, for otherwise $\ell\left(c^{\prime}\right) \geq(5,6,8)$ or $(6,6,7)$. Let $d_{1}, d_{2}$ denote the out-vertices on $C_{i-3}$ such that $R\left(d_{1}\right)=$ $L\left(d_{2}\right)=B\left(c_{1}\right)$. Since $\left|R\left(c^{\prime}\right)\right|=5, c^{\prime}$ is adjacent to $d_{2}$ and $\left|R\left(c^{\prime}\right) \cap C_{i-3}\right|=3$. Further we have $\left|B\left(d_{2}\right)\right| \geq 6$. Since $i-3 \geq 18$ and by (5.4), $C_{i-3}\left(d_{1}, d_{2}\right) \neq \emptyset$. Since $\left|L\left(c^{\prime}\right)\right| \leq 7$, we may assume $d$ is the only vertex in $C_{i-3}\left(d_{1}, d_{2}\right)$. Then $|R(d)|=\left|B\left(d_{2}\right)\right| \geq 6$, and $|L(d)|=\left|B\left(d_{1}\right)\right| \geq 5$. In fact, $|L(d)|=5$ and $|R(d)|=6$, for otherwise $\ell(d) \geq(6,6,7)$ or $(5,7,7)$. Thus $\left|L(d) \cap C_{i-4}\right|=2=\left|R(d) \cap C_{i-4}\right|$. Hence $C_{i-4}$ has three consecutive out-vertices. Since $i-4 \geq 17$, this is a contradiction to (5.4).

(6.3) Lemma. Let $i \geq 24$, let $a_{1}$ and $a_{2}$ be consecutive in-vertices on $C_{i}$ such that (i) $R\left(a_{1}\right)=L\left(a_{2}\right)$ and (ii) $\left|C_{i}\left(a_{1}, a_{2}\right)\right|=2$. Let $b_{1}, b_{2}$ denote the out-vertices on $C_{i-1}$ such that $R\left(b_{1}\right)=L\left(b_{2}\right)=R\left(a_{1}\right)$. Suppose $\left|C_{i-1}\left(b_{1}, b_{2}\right)\right| \geq 2$. Let $b_{3}, b_{4}$ be the vertices in $C_{i-1}\left(b_{1}, b_{2}\right)$ such that $C_{i-1}\left(b_{1}, b_{3}\right)=\emptyset=C_{i-1}\left(b_{4}, b_{2}\right)$. Then both $b_{3}$ and $b_{4}$ are outvertices.

Proof. Suppose this is false. By symmetry, assume that $b_{3}$ is an in-vertex. Since $\left|C_{i-1}\left(b_{1}, b_{2}\right)\right| \geq 2,\left|A\left(b_{3}\right)\right|=\left|R\left(b_{1}\right)\right|=\left|L\left(b_{2}\right)\right| \geq 8$. Hence $b_{3}$ is adajcent to $C_{i-2}$; otherwise, $\left|L\left(b_{3}\right)\right| \geq 6$ and $\left|R\left(b_{3}\right)\right| \geq 5$, and so, $\ell\left(b_{3}\right) \geq(5,6,8)$, a contradiction.

Let $c$ denote the neighbor of $b_{3}$ on $C_{i-2}$. We consider two cases.

Case 1. $\left|R\left(b_{3}\right)\right|=4$.

Let $c_{2}$ be the out-vertex on $C_{i-2}$ such that $L\left(c_{2}\right)=R(c)$. See Figure 23(a). Since $\left|R\left(b_{3}\right)\right|=4, C_{i-2}\left(c, c_{2}\right)=\emptyset$. Let $c_{1}^{\prime}, c_{2}^{\prime}$ denote the in-vertices on $C_{i-2}$ such that $R\left(c_{1}^{\prime}\right)=$ $B(c)=L\left(c_{2}^{\prime}\right)$, and let $d_{1}, d_{2}$ be the out-vertices on $C_{i-3}$ such that $R\left(d_{1}\right)=B(c)=L\left(d_{2}\right)$. Since $i-2 \geq 22$ and by $(6.1)$ and (6.2), $\left|C_{i-3}\left(d_{1}, d_{2}\right)\right| \geq 3$. Hence $|B(c)|=\left|B\left(c_{2}\right)\right| \geq 8$. By (5.4), $C_{i-2}\left(c_{1}^{\prime}, c\right)=\emptyset=C_{i-2}\left(c_{2}, c_{2}^{\prime}\right)$. So $\left|B\left(b_{1}\right)\right|=\left|A\left(c_{1}^{\prime}\right)\right| \geq 6$ and $\left|R\left(c_{1}^{\prime}\right)\right|=|B(c)| \geq 8$. Hence $\left|L\left(c_{1}^{\prime}\right)\right|=4$, as otherwise $\ell\left(c_{1}^{\prime}\right) \geq(5,6,8)$. Therefore $c_{1}^{\prime}$ is adjacent to $d_{1}$. Note $\left|L\left(d_{1}\right)\right|=\left|L\left(c_{1}^{\prime}\right)\right|=4$ and $\left|R\left(d_{1}\right)\right|=\left|R\left(c_{1}^{\prime}\right)\right| \geq 8$. Since $i-3 \geq 21$ and since $L\left(d_{1}\right) \cap C_{i-3}$ consists of two consecutive out-vertices on $C_{i-3}$, it follows from (6.1) and (6.2) that $\left|B\left(d_{1}\right)\right| \geq 8$. Thus $\ell\left(d_{1}\right) \geq(4,8,8)$, a contradiction.

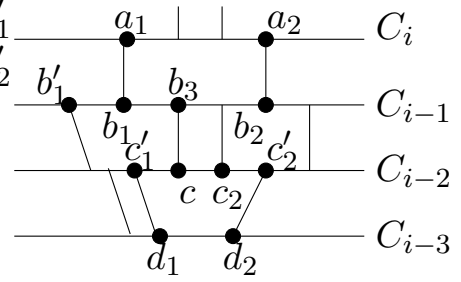

(a)

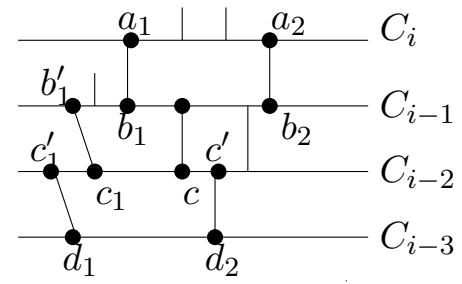

Figure 23: Proof of Lemma (6.3) 
Case 2. $\left|R\left(b_{3}\right)\right| \geq 5$.

In this case, $\left|L\left(b_{3}\right)\right| \geq 5 \leq\left|R\left(b_{3}\right)\right|$. So $\left|L\left(b_{3}\right)\right|=\left|R\left(b_{3}\right)\right|=5$, for otherwise, $\ell\left(b_{3}\right) \geq$ $(5,6,8)$. Also $\left|R\left(b_{1}\right)\right| \leq 9$, or else $\ell\left(b_{3}\right) \geq(5,5,10)$.

Let $b_{1}^{\prime}$ be the in-vertex on $C_{i-1}$ such that $R\left(b_{1}^{\prime}\right)=B\left(b_{1}\right)$, and let $c_{1}, c$ be the outvertices on $C_{i-2}$ such that $R\left(c_{1}\right)=L(c)=B\left(b_{1}\right)$. See Figure 23(b). Since $\left|L\left(b_{3}\right)\right|=5$, $C_{i-2}\left(c_{1}, c\right)=\emptyset, b_{3}$ is adjacent to $c$, and $b_{1}^{\prime}$ is adjacent to $c_{1}$.

Let $c_{1}^{\prime}, c^{\prime}$ be the in-vertices on $C_{i-2}$ such that $R\left(c_{1}^{\prime}\right)=L\left(c^{\prime}\right)=B\left(c_{1}\right)$. Since $i-2 \geq 22$ and by (5.4), $C_{i-2}\left(c_{1}^{\prime}, c_{1}\right)=\emptyset=C_{i-2}\left(c, c^{\prime}\right)$. Since $i-3 \geq 21$ and by (6.1) and (6.2), $\left|C_{i-3}\left(d_{1}, d_{2}\right)\right| \geq 2$. Hence $\left|B\left(c_{1}\right)\right|=|B(c)|=\left|L\left(c^{\prime}\right)\right| \geq 8$.

Let $d_{1}, d_{2}$ denote the out-vertices on $C_{i-3}$ such that $R\left(d_{1}\right)=L\left(d_{2}\right)=B\left(c_{1}\right)$. Since $\left|R\left(b_{3}\right)\right|=5, b_{3}$ is adjacent to $c$, and $C_{i-2}\left(c, c^{\prime}\right)=\emptyset$, we have $\left|R(c) \cap C_{i-2}\right|=3$. Thus $c^{\prime}$ is adjacent to $d_{2}$, for otherwise, $\ell\left(c^{\prime}\right) \geq(5,6,8)$. So $\left|R\left(d_{2}\right)\right| \geq 5$. Further, if $\left|R\left(d_{2}\right)\right|=5$, then $\left|B\left(d_{2}\right)\right| \geq 6$. Therefore, $\ell\left(d_{2}\right) \geq(5,6,8)$, a contradiction.

(6.4) Lemma. Let $i \geq 24$, let $a_{1}$ and $a_{2}$ be consecutive in-vertices on $C_{i}$ such that $R\left(a_{1}\right)=L\left(a_{2}\right)$. Then $\left|C_{i}\left(a_{1}, a_{2}\right)\right| \leq 1$.

Proof. Suppose $\left|C_{i}\left(a_{1}, a_{2}\right)\right| \geq 2$. Then by (5.4), $\left|C_{i}\left(a_{1}, a_{2}\right)\right|=2$. Let $b_{1}, b_{2}$ denote the out-vertices on $C_{i-1}$ such that $R\left(b_{1}\right)=L\left(b_{2}\right)=R\left(a_{1}\right)$. See Figure 24. By (6.1) and (6.2), $\left|C_{i-1}\left(b_{1}, b_{2}\right)\right| \geq 2$ and $\left|R\left(b_{1}\right)\right|=\left|L\left(b_{2}\right)\right| \geq 8$. Let $b_{3}, b_{4}$ be the vertices in $C_{i-1}\left(b_{1}, b_{2}\right)$ such that $C_{i-1}\left(b_{1}, b_{3}\right)=\emptyset=C_{i-1}\left(b_{4}, b_{2}\right)$. By $(6.3)$, both $b_{3}$ and $b_{4}$ are out-vertices. Let $c_{1}, c$ be the out-vertices on $C_{i-2}$ such that $R\left(c_{1}\right)=B\left(b_{1}\right)=L(c)$. Since $i-1 \geq 23$, it follows from (6.1) and (6.2) that $\left|C_{i-2}\left(c_{1}, c\right)\right|=0$. Hence $\left|B\left(b_{1}\right)\right|=\left|B\left(b_{3}\right)\right| \geq 8$.

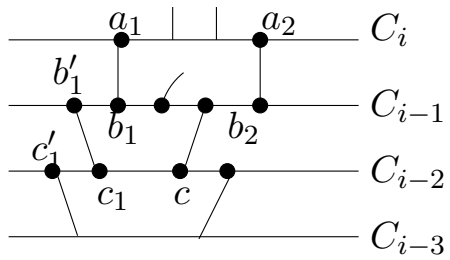

Figure 24: Proof of Lemma (6.4)

By $(5.4), C_{i-1}\left(b_{1}^{\prime}, b_{1}\right)=\emptyset$. Thus $\left|L\left(b_{1}\right)\right| \geq 4$. Therefore $\ell\left(b_{1}\right) \geq(4,8,8)$, a contradiction.

\section{The proof of the main result}

In this section, we complete the proof of (1.2). Let $G$ be a positively curved cubic plane graph such that $G$ is locally finite and every face of $G$ is boudned by a cycle. 
Assume that $G$ is infinite. Then $G$ has a nice sequence $\left(C_{0}, C_{1}, \ldots\right)$. By $(2.4)$, we may assume that $G$ is nicely embedded with respect to $\left(C_{0}, C_{1}, \ldots\right)$.

(7.1) Lemma. For $i \geq 26,\left|C_{i-1}\right|>\left|C_{i}\right|$.

Proof. Let $a_{1}, a_{2}, \ldots, a_{n}$ denote the in-vertices on $C_{i}$ and occur on $C_{i}$ in that clockwise order. For each $j \in\{1, \ldots, n\}$, let $b_{j}, b_{j}^{\prime}$ be the out-vertices on $C_{i-1}$ such that $R\left(b_{j}\right)=$ $R\left(a_{j}\right)$ and $L\left(b_{j}^{\prime}\right)=L\left(a_{j}\right)$. See Figure 25 . For convenience, let $b_{n+1}=b_{1}, b_{n+1}^{\prime}=b_{1}^{\prime}$, and $a_{n+1}=a_{1}$.
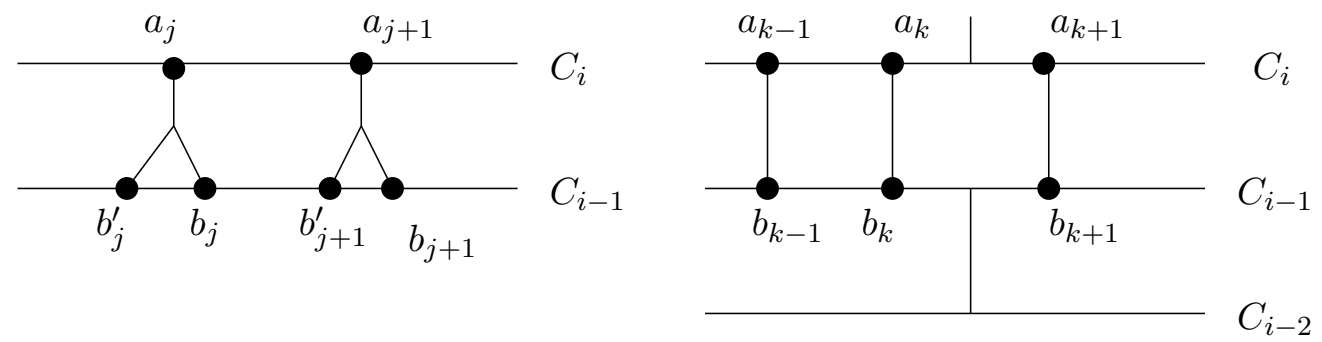

Figure 25: Proof of Lemma (7.1)

To prove the lemma, it suffices to show that for each $j \in\{1, \ldots, n\},\left|C_{i-1}\left(b_{j}, b_{j+1}^{\prime}\right)\right| \geq$ $\left|C_{i}\left(a_{j}, a_{j+1}\right)\right|$, and there is some $k$ such that $\left|C_{i-1}\left(b_{k}, b_{k+1}^{\prime}\right)\right|>\left|C_{i}\left(a_{k}, a_{k+1}\right)\right|$.

By (6.4), $\left|C_{i}\left(a_{j}, a_{j+1}\right)\right| \leq 1$.

If $\left|C_{i}\left(a_{j}, a_{j+1}\right)\right|=0$, then clearly $\left|C_{i-1}\left(b_{j}, b_{j+1}^{\prime}\right)\right| \geq\left|C_{i}\left(a_{j}, a_{j+1}\right)\right|$. Now assume that $\left|C_{i}\left(a_{j}, a_{j+1}\right)\right|=1$. Since $i-1 \geq 24$, it follows from (6.4) that $\left|C_{i-1}\left(b_{j}, b_{j+1}^{\prime}\right)\right| \geq 1$. So $\left|C_{i-1}\left(b_{j}, b_{j+1}^{\prime}\right)\right| \geq\left|C_{i}\left(a_{j}, a_{j+1}\right)\right|$.

Hence we may assume that $b_{j}=b_{j}^{\prime}$ for all $j \in\{1, \ldots, n\}$, for otherwise, we have $\left|C_{i-1}\right|>\left|C_{i}\right|$. Because $G$ is connected and $\left(C_{0}, C_{1}, \ldots\right)$ is an infinite sequence, there is some $k \in\{1, \ldots, n\}$ such that $\left|C_{i}\left(a_{k}, a_{k+1}\right)\right|=1$ for some $k$. Now $\left|B\left(b_{k}\right)\right| \geq 5$.

If $\left|B\left(b_{k}\right)\right|=5$, then $\left|B\left(b_{k}\right) \cap C_{i-2}\right|=2$, contradicting (6.4) (because $i-2 \geq 24$ ). So $\left|B\left(b_{k}\right)\right| \geq 6$. Hence $\left|L\left(b_{k}\right)\right| \leq 5$, or else $\ell\left(b_{k}\right) \geq(6,6,6)$. In fact, $\left|L\left(b_{k}\right) \cap C_{i-1}\right| \geq 3$ by (6.4) (since $i-1 \geq 25)$. So $\left|C_{i-1}\left(b_{k-1}, b_{k}^{\prime}\right)\right|=\left|C_{i-1}\left(b_{k-1}, b_{k}\right)\right|>\left|C_{i}\left(a_{k-1}, a_{k}\right)\right|$. Therefore, $\left|C_{i-1}\right|>\left|C_{i}\right|$.

It is now easy to see that (1.2) holds because of the contradiction caused by (7.1) and the infinite sequence $\left(C_{0}, C_{1}, \ldots\right)$.

\section{References}

[1] M. Gromov, Hyperbolic groups in Essays in Group Theory (S. M. Gersten, editor) M.S.R.I. Publications (1987) 75-263. 
[2] Y. Higuchi, Combinatorial curvature for planar graphs, J. Graph Theory 38 (2001) 220-229.

[3] S. B. Myers, Riemannian manifolds with positive mean curvature, Duke Math. J. 8 (1941) 401-404. 